\title{
A Comparison Principle for Singular Diffusion Equations with Spatially Inhomogeneous Driving Force for Graphs
}

\author{
M.-H. Giga, Y. Giga \& P. RybKa
}

Communicated by F. Otto

\begin{abstract}
We introduce the notions of viscosity super- and subsolutions suitable for singular diffusion equations of non-divergence type with a general spatially inhomogeneous driving term. In particular, the viscosity super- and subsolutions support facets and allow a possible facet bending. We prove a comparison principle by a modified doubling variables technique. Finally, we present examples of viscosity solutions. Our results apply to a general crystalline curvature flow with a spatially inhomogeneous driving term for a graph-like curve.
\end{abstract}

\section{Introduction}

As a continuation of $[17,21]$ this paper studies a degenerate nonlinear parabolic equation (in one space dimension) whose diffusion effect is very strong at particular slopes of unknown functions. We are particularly interested in an equation, where the driving force term is spatially inhomogeneous. A typical example, which we have in mind, is a quasilinear equation

$$
u_{t}=a\left(u_{x}\right)\left[W^{\prime}\left(u_{x}\right)_{x}+\sigma(t, x)\right],
$$

where $W$ is a given convex function on $\mathbf{R}$ but $W$ may not be of class $C^{1}$ so that its derivative $W^{\prime}$ may have jump discontinuities. Here $a$ is a given non-negative continuous function and $\sigma$ is a given smooth function depending on $x$ and also on $t$, where $u_{t}$ and $u_{x}$ denote the time and the space derivative of $u=u(t, x)$.

As explained in detail in [17] the equation is viewed as an evolution law of the graph of $u$ moved by an anisotropic mean curvature flow $V=M(\mathbf{n})\left(\kappa_{\gamma}+\sigma\right)$ with a singular interfacial energy density $\gamma$, where $\kappa_{\gamma}$ is a weighted curvature and $M$ is mobility; $V$ denotes the normal velocity of the evolving curve in the direction of $\mathbf{n}$ (the quantity $\kappa_{\gamma}$ formally equals $\left(\gamma^{\prime \prime}+\gamma\right) \kappa$ with curvature $\kappa$ and $\gamma=\gamma(\theta)$ is an interfacial density as a function of the argument $\theta$ of $\mathbf{n}=(\cos \theta, \sin \theta))$. 
Our eventual goal is to establish a kind of the theory of viscosity solutions for a class of equations including (1.1) as a particular example so that we are able to construct a global-in-time solution, for instance for periodic initial data. In this paper we give a new notion of viscosity solutions for (1.1) and we establish a comparison principle.

If $\sigma$ in (1.1) is independent of $x$, the theory of viscosity solutions has been already established in $[17,21]$. Even in this simpler case the quality $\left(W^{\prime}\left(u_{x}\right)\right)_{x}$ turns to be nonlocal so the conventional viscosity theory does not work. For example if $W(p)=|p|$, then $W^{\prime \prime}(p)$ is two times the delta function so that (1.1) becomes

$$
u_{t}=a\left(u_{x}\right)\left[2 \delta\left(u_{x}\right) u_{x x}+\sigma(t)\right]
$$

which is, of course, not a classical partial differential equation. If $u=u(t, x)$ has a flat part (called a facet) with a zero slope, then it is expected to move with speed $u_{t}=a(0)[2 \chi / L+\sigma]$ provided that a facet persists and it does not break. Here $L$ is the length of a facet (which is a nonlocal quantity) and $\chi= \pm 1,0$ is a transition number of the facet depending upon local behavior of $u$ near the facet. For example, if $u$ is 'concave' near the facet, then $\chi$ should be -1 . When $\sigma$ is spatially homogeneous, the hypothesis that a facet does not break is justified either by the viscosity theory developed by $[17,20]$ or by the subdifferential theory [14] (in the case $\sigma \equiv 0$ ), in the sense that such a solution is an appropriate limit of solutions to strictly parabolic problems. When $W$ is piecewise linear and $\sigma$ is independent of $x$, then $(1.1)$ is analyzed in $[1,38]$ for a very restrictive class of unknown functions, which are piecewise linear, with slopes belonging to jump discontinuities of $W$. Their 'admissible' solution is actually a solution in a viscosity sense [17] and also in a variational sense $[12,14]$.

If $\sigma$ depends on the space variable, the hypothesis that all facets do not break is no longer true. For example, if we postulate this hypothesis, then the speed, $u_{t}$, of a facet with the slope equal to zero when $u$ is a solution of (1.2) is $a(0)[2 \chi / L+f \sigma \mathrm{d} x]$, where $f$ denotes the average over the facet. As noticed in [19], if we assigned the speed in this way the solution may not in general enjoy the comparison principle. This shows that such a 'solution' is not obtained as a limit of approximate problems satisfying the comparison principle. On the other hand if $\left|\sigma_{x}\right|$ is sufficiently small compared with the length of facets, such a solution is known to enjoy a comparison principle [3].

If $a$ is a constant, say $a \equiv 1$, and $\sigma$ is independent of $t,(1.1)$ can be viewed as a subdifferential formulation

$$
u_{t} \in-\partial \varphi(u)
$$

where $\varphi$ is an energy which formally equals

$$
\varphi(u)=\int_{\mathbf{T}}\left[W\left(u_{x}\right)-\sigma(x) u\right] \mathrm{d} x ;
$$

for simplicity, we assume here a periodic boundary condition so that $\mathbf{T}=\mathbf{R} / \omega \mathbf{Z}$. As observed in [18] for (1.3), a general theory of subdifferential equations in the Hilbert space $L^{2}(\mathbf{T})$ provides not only the unique existence of the solution but also 
the value of right derivative $\mathrm{d} u^{+} / \mathrm{d} t$ (of $u$ as a function with value in $H$ ). A general theory further yields

$$
\mathrm{d}^{+} u / \mathrm{d} t=-\partial^{0} \varphi(u)
$$

where $\partial^{0} \varphi$ is the canonical restriction of a closed convex set $\partial \varphi(u(t))$, that is

$$
\partial^{0} \varphi(u)=\arg \min \left\{\|f\|_{H} \mid f \in \partial \varphi(u) \subset H\right\} .
$$

In [18], it is observed that $\partial^{0} \varphi$ can be calculated by solving an obstacle problem. Let us review those observations. Since the condition

$$
f \in-\partial \varphi(u)
$$

is equivalent to

$$
f(x)=\eta_{x}(x)+\sigma(x), \eta(x) \in \partial W\left(u_{x}(x)\right), \text { almost every where } x \in \mathbf{T},
$$

the quantity

$$
-\partial^{0} \varphi(u)=\left\{\begin{array}{lll}
\left(W^{\prime}\left(u_{x}(x)\right)\right)_{x}+\sigma(x) & \text { if } & u_{x} \notin P \\
\eta_{x}^{0}(x)+\sigma(x) & \text { if } & u_{x} \in P
\end{array}\right.
$$

where $P$ is the jump discontinuity of $W^{\prime}$ and $u$ is assumed to be of class $C^{2}$ and $P$-faceted [17]. Here, $\eta^{0}$ minimizes

$$
\left\{\int_{F}\left|\eta_{x}+\sigma\right|^{2} \mathrm{~d} x ; \eta \in \partial W\left(u_{x}(x)\right)\right\}
$$

under a suitable boundary condition at the end of facet $F$ depending on whether $u$ is 'convex' or 'concave' near $F$. This is a convex minimizing problem so a unique minimizer always exists. Moreover, if $\sigma$ is independent of $x, \eta_{x}$ must be constant and $\eta_{x}^{0}+\sigma=\chi / L+\sigma$. If $\sigma$ depends on $x, \eta_{x}^{0}+\sigma$ may not be a constant over $F$ and this is one reason why the speed may not be a constant on $F$ when $\sigma$ depends on $x$. The subdifferential equation (1.3) can be approximated by a smooth parabolic problem, so we expect the comparison principle to hold. Thus, it is natural to guess that $\eta_{x}^{0}+\sigma$ gives a candidate for the value of

$$
\left.\Lambda_{W}^{\sigma}(u)(x)\right)=\left(W^{\prime}\left(u_{x}\right)\right)_{x}+\sigma(x)
$$

when $W^{\prime}$ has jump discontinuities. Note that this quantity agrees with the minimal velocity profile proposed by [36], as observed in [18].

Unfortunately, a general equation (1.1) cannot be viewed as a subdifferential equation (1.3). However, we still use (1.4) to define (1.6). We establish a notion of viscosity solutions by assigning the value $\Lambda_{W}^{\sigma}$ by (1.4) for test functions which we call admissible. The class of test functions is the same as [17] so a facet of a test function never vanishes or breaks. The idea of the proof of the comparison principle is similar to that of [17] except for a simplified handling of end points of facets observed by [21] and the use of continuity of $\Lambda_{W}^{\sigma}(u)$ under the translation 
of a faceted region which is obvious when $\sigma$ is constant. So we have to study an obstacle problem in this paper carefully. Let $\Lambda(F)(x)$ be a quantity defined by

$$
\Lambda(F)(x)=\eta_{x}^{0}(x)+\sigma(x), \quad x \in F
$$

where $\eta^{0}$ is the minimizer of (1.5). In particular, we prove that

$$
\Lambda\left(F^{\mu}\right)(x-\mu) \rightarrow \Lambda(F)(x)
$$

as $\mu \rightarrow 0$, where $F^{\mu}=F-\mu=\{x \mid x+\mu \in F\}$ provided that $\sigma_{x}$ is bounded. Moreover, the convergence is uniform with respect to $F$ provided that $F$ is bounded. This problem can be viewed as a stability problem for (1.5) with respect to perturbations of $\sigma$. Since our obstacle problem is convex, it is not difficult to prove these facts. We also need comparison results (maximum principle) for $\Lambda_{W}^{\sigma}$ to see that this quantity behaves like curvature or usual second derivatives. It is often convenient to consider $\xi=\eta+\int^{x} \sigma$ as a variable, instead of $\eta$ itself, so we shall use variable $\xi$. We warn the reader that in Section 5 we will use differently defined $\xi$.

To establish the comparison principle we argue by contradiction using the doubling variables technique. Let $u$ be a subsolution and $v$ be a supersolution. We are interested in the maximizers of

$$
u(t, x)-v(s, y)-B_{\varepsilon}(x-y)-(t-s)^{2} / \delta-\gamma /(T-t)-\gamma /(T-s)
$$

for small $\varepsilon, \delta, \gamma>0$. Here $B_{\varepsilon}=\varepsilon B(x / \varepsilon), B(x) \backsim x^{2}$ for large $x$ and $B$ is a (non-negative) faceted $C^{2}$ convex function with $B(0)=0$. This choice of a test function $B$ is different from [17] and this choice simplifies the argument. We use sup-convolutions with a faceted function to regularize the problem as in [17]. Quantity $\Lambda_{W}^{\sigma}$ behaves like a usual second derivative in the sense that it satisfies the maximum principle. At the final stage we have to compare $\Lambda\left(F^{\mu}\right)$ and $\Lambda(F)$ which is trivial when $\sigma$ is constant, because it is independent of $\mu$.

Although this paper focuses on the comparison principle for (1.1), as observed in [21], the method developed here is fundamental to establish a level set method for $V=M(\mathbf{n})\left(\kappa_{\gamma}+\sigma\right)$ when $\sigma$ depends on $x$. For a standard level set method for smooth $\gamma$ see $[10,13,16]$. Also a stability result is expected [20] but we do not intend to include any progress in this direction in the present paper. A general existence result through Perron's method is almost the same as the one in [17], though we do not state it explicitly. Instead, we give a couple of examples of solutions. An existence result based on Perron's method and using the comparison principle established in the present paper was established in [25].

Recently, besides examples in [18], several semi-explicit variational solutions are constructed for (1.1) for special choices of $M, \sigma$ and $\gamma$ by solving a free boundary problem $[27,29,30]$. Their variational solutions are expected to be our viscosity solutions. In this paper we shall confirm this consistency at least for some typical examples.

We do not know much about surface evolutions. In surface evolving problems a facet may not stay as a facet even if $\sigma \equiv 0$ see for example [5-8].

After this paper was submitted, we were informed of a very recent work [9] by Chambolle and Novaga, where they established a local-in-time unique solution for a closed curve with spatially inhomogeneous $\sigma$. 
A notion of a generalized solution is established and a comparison principle is proved in [4], see also [3]. However, the existence of a solution is known only when the initial surface is convex, see [2]; note that their problem is formulated as $V=\gamma \kappa_{\gamma}$ where the mobility parallels the interfacial energy.

The bibliographies of review papers [15,22-24] include several articles dealing with anisotropic curvature flow equations with singular interfacial energy or singular diffusion equations. Here, we only mention a few recent works related to this topic but not included in the papers mentioned above. In particular, we have in mind the approach developed by MucHA and RYBKA, which is based on an original definition of a composition of multivalued operators, see [32,34]. So far, it is restricted to one dimension but allows one to study facet evolution for quite general data as well as the regularity of solutions.

This paper is organized as follows. We first study an obstacle problem in Section 2. In Section 3, we establish a notion of viscosity solutions. In Section 4, we prove our main comparison theorem. In Section 5 we shall prove that the semiexplicit solutions in [29] are indeed solutions in our viscosity sense.

\section{Variational Properties of Nonlocal Curvature with a Nonuniform Driving Force Term}

We shall give a variational characterization of the quantity $\Lambda_{W}^{\sigma}$, which is formally defined by

$$
\Lambda_{W}^{\sigma}(u)(x)=\left(W^{\prime}\left(u_{x}\right)\right)_{x}+\sigma(x)
$$

by means of solving an obstacle problem. This characterization enables us to derive various important properties to establish the theory of viscosity solutions for singular diffusion equations.

\subsection{An Obstacle Problem}

Let $Z$ be a real-valued $C^{2}$ (or $C^{1,1}$ ) function, defined in a bounded interval $\bar{I}$, where $I=(a, b)$. For a given $\Delta>0$ let $K_{\chi_{l} \chi_{r}}^{Z}$ be the set of all $\xi \in H^{1}(I)$ satisfying

$$
Z(x)-\Delta / 2 \leqq \xi(x) \leqq Z(x)+\Delta / 2 \text { for } x \in I \text { (obstacle condition) }
$$

and

$$
Z(x)-\Delta / 2 \leqq \xi(x) \leqq Z(x)+\Delta / 2 \text { for } x \in I \text { (obstacle condition). }
$$

Here, $\chi_{l}$ and $\chi_{r}$ take values \pm 1 . Let $J_{\chi_{l} \chi_{r}}^{Z}$ be the functional on $L^{2}(I)$ defined by

$$
J_{\chi_{l} \chi_{r}}^{Z}(\xi)= \begin{cases}\int_{a}^{b}\left|\xi^{\prime}(x)\right|^{2} \mathrm{~d} x, & \xi \in K_{\chi_{l} \chi_{r}}^{Z} \\ \infty, & \text { otherwise. }\end{cases}
$$

In this subsection, we suppress the dependence with respect to $Z$ since we fix $Z$. By the definition of $J_{\chi_{l} \chi_{r}}$, it is easy to see that inf $J_{\chi_{l} \chi_{r}}$ is the $H^{1}$-homogeneous 
distance from zero to the convex closed set $K_{\chi_{l} \chi_{r}}$ in $H^{1}$. Thus, $J_{\chi_{l} \chi_{r}}$ admits a unique absolute minimizer denoted by $\xi_{\chi_{l} \chi_{r}}$. Evidently, $\xi_{\chi_{l} \chi_{r}} \in H^{1}(I) \subset C^{1 / 2}(\bar{I})$ by the Sobolev embedding. In fact, it is $C^{1,1}$, as proved in [33, Chap II, Theorem 7.1] (in [33], the regularity of the multidimensional obstacle problem is also discussed). In our one-dimensional case, as discussed below, it is easy to prove that $\xi_{\chi_{l} \chi_{r}}$ is $C^{1,1}$ since the obstacle is $C^{1,1}$ and the coincidence set is closed.

For $\xi \in H^{1}(I)$ let $D_{ \pm}(\xi)$ be the coincidence set defined by

$$
D_{ \pm}=D_{ \pm}(\xi)=\{x \in \bar{I} \mid \xi(x)=Z(x) \pm \Delta / 2\} .
$$

We say that $D_{+}$is the upper coincidence set while $D_{-}$is the lower coincidence set.

Definition 1. We say that $\xi \in K_{\chi_{l} \chi_{r}}$ satisfies the concave-convex condition if $\xi$ is concave on each connected component of the complement of the upper coincidence set $D_{+}$and convex on each connected component of the complement of the lower coincidence set $D_{-}$, that is, $\xi^{\prime \prime} \leqq 0$ outside $D_{+}$and $\xi^{\prime \prime} \geqq 0$ outside $D_{-}$. In particular, $\xi$ is $C^{1,1}$ in $I$ and $\xi^{\prime \prime}=0$ outside $D_{-} \cup D_{+}$.

Proposition 1 (A characterization of the minimizer). The function $\xi \in K_{\chi_{l} \chi_{r}}$ is the minimizer of $J_{\chi_{l} \chi_{r}}$ if and only if $\xi$ fulfills the concave-convex condition. In particular, $\xi_{\chi_{l} \chi_{r}}$ is $C^{1,1}$ in I and

$$
\sup _{x \in I}\left|\xi_{\chi_{l} \chi_{r}}^{\prime \prime}(x)\right| \leqq \sup _{x \in I}\left|Z^{\prime \prime}(x)\right| .
$$

Proof. By the convexity of $J_{\chi_{l} \chi_{r}}$ and the uniqueness of the minimizer, $\xi \in K_{\chi_{l} \chi_{r}}$ is the absolute minimizer if and only if $\xi$ is a local minimizer of $J_{\chi_{l} \chi_{r}}$ that is,

$$
\int_{D_{+}^{c}} \xi^{\prime} \varphi^{\prime} \mathrm{d} x \geqq 0, \int_{D_{-}^{c}} \xi^{\prime} \psi^{\prime} \mathrm{d} x \leqq 0
$$

for all $\varphi \in H^{1}(I)$ satisfying $\varphi(a)=\varphi(b)=0$ and $\varphi \geqq 0$ in $D_{+}^{c}=\bar{I} \backslash D_{+}$and for all $\psi \in H^{1}(I)$ satisfying $\psi(a)=\psi(b)=0$ and $\psi \geqq 0$ in $D_{-}^{c}=\bar{I} \backslash D_{-}$ by the obstacle condition (2.2) and the boundary condition (2.3). These conditions are equivalent to the concave-convex condition. We refer to ScHWARTz [37] or HÖRMANDER [31] for the equivalence of convexity in the distribution sense and the strong convexity.

The remaining statement is a simple consequence of the concave-convexity condition.

As a trivial application we give two cases, where the minimizer is explicitly written.

Corollary 1. (i) If the concave hull $Z_{\text {cave }}$ of $Z$ in I is smaller than $Z+\Delta$, that is, $Z_{\text {cave }} \leqq Z+\Delta$ in $I$, then $\xi_{+-}=Z_{\text {cave }}-\Delta / 2$.

(ii) If the straight line function $\xi(x)=\xi(a)+(Z(b)-Z(a)+\Delta)(x-a) /(b-a)$ fulfills the obstacle condition (2.2), then it is the minimizer of $J_{++}$provided that $\xi(a)=Z(a)-\Delta / 2$ and $\xi(b)=Z(b)+\Delta / 2$. Here, $J_{++}=J_{\chi_{l} \chi_{r}}$ when $\chi_{l}=\chi_{r}=1$. 


\subsection{Comparison Principle}

So far, we have fixed interval $I$ to define $\xi_{\chi_{l} \chi_{r}}$. We shall study the dependence of $\xi_{\chi_{l} \chi_{r}}^{\prime}$ upon $I$. To clarify this, we write $J_{\chi_{l} \chi_{r}, I}$ instead of $J_{\chi_{l} \chi_{r}}^{Z}$ and $\xi_{\chi_{l} \chi_{r}, I}$ instead of $\xi_{\chi_{l} \chi_{r}}^{Z}$. We set

$$
\Lambda_{\chi_{l} \chi_{r}}^{Z^{\prime}}(x, I)=\frac{\mathrm{d} \xi_{\chi_{l} \chi_{r}, I}(x)}{\mathrm{d} x}
$$

It is easy to observe that this quantity agrees with $\eta_{x}^{0}+\sigma$ when $Z$ equals a primitive of $\sigma$. It is sufficient to take $\xi=\eta+Z$. The reason we write $Z^{\prime}$ instead of $Z$ is that the derivative of $\xi_{\chi_{l} \chi_{r}}^{Z}$ depends on $Z$ only through its derivative. We suppress $Z^{\prime}$ in (2.5) when we fix $Z$. We shall write $\Lambda_{-+}$etc. instead of writing $\Lambda_{\{-1\},\{+1\}}$.

Theorem 1. (Comparison principle) Assume that $I_{1}$ and $I_{2}$ are bounded open intervals.

(i) If $I_{2} \subset I_{1}$, then

$$
\Lambda_{--}\left(x, I_{2}\right) \leqq \Lambda_{ \pm \pm}\left(x, I_{1}\right) \leqq \Lambda_{++}\left(x, I_{2}\right) \text { for } x \in I_{2} .
$$

(ii) If $a \leqq c<b \leqq d$ for $I_{1}=(a, b), I_{2}=(c, d)$, then for $x \in(c, b)$

$$
\Lambda_{ \pm-}\left(x, I_{1}\right) \leqq \Lambda_{+ \pm}\left(x, I_{2}\right), \Lambda_{- \pm}\left(x, I_{2}\right) \leqq \Lambda_{ \pm+}\left(x, I_{1}\right) .
$$

This can be proved by a comparison principle for parabolic equations by an approximation, as is done in Giga-Gurtin-Matias [28]. However, since the problem is one dimensional, we give instead an elementary proof, which is based on the following simple observation.

Lemma 1. (Elementary Lemma) Let us suppose that $\varphi, \psi \in C^{1,1}([a, b])$ are such that $\psi \leqq \varphi$. Functions $\xi, \zeta \in H^{1}(a, b)$ are minimizers of

$$
\int_{a}^{b}\left|\frac{\mathrm{d} u}{\mathrm{~d} x}\right|^{2} \mathrm{~d} x
$$

satisfying the following constraints $\psi \leqq u \leqq \varphi$ and the boundary conditions,

$$
\xi(a)=A=\zeta(a), \quad \xi(b)=B>B^{\prime}=\zeta(b) .
$$

Then $\xi^{\prime}(x) \geqq \zeta^{\prime}(x)$ almost everywhere in $(a, b)$.

Proof. It is very easy to see that $\xi(x) \geqq \zeta(x)$ for $x \in[a, b]$. We have to show that function $\xi-\zeta$ is increasing. For this purpose we will see that $\xi-\zeta$ may attain neither local maximum nor minimum in $(a, b)$. In fact, the lack of local maxima implies impossibility of local minima. Thus it is sufficient to see that no local maximum of $\xi-\zeta$ is possible.

Let us suppose the contrary, that is, there exists a point $x_{0} \in(a, b)$ where $\xi-\zeta$ attains a local maximum, that is there is a positive $\delta$, such that $\left(x_{0}-\delta, x_{0}+\delta\right) \subset$ $[a, b]$ and

$$
(\xi-\zeta)\left(x_{0}\right) \geqq(\xi-\zeta)(x) \quad \text { for } \quad x \in\left(x_{0}-\delta, x_{0}+\delta\right),
$$


where the inequality is strict for $x=x_{0} \pm \delta$. We will consider a number of cases upon the lower coincidence set

$$
D_{-}(\zeta)=\{x \in[a, b] \mid \zeta(x)=\psi(x)\}
$$

They are:

(i) $\quad\left(x_{0}-\delta, x_{0}+\delta\right) \subset\left(D_{-}(\zeta)\right)^{c}$, that is $\zeta$ is a line segment over $\left(x_{0}-\delta, x_{0}+\delta\right)$;

(ii) $\quad\left(x_{0}-\delta, x_{0}+\delta\right) \subset D_{-}(\zeta)$;

(iii) $x_{0} \in \overline{D_{-}(\zeta)} \cap \overline{\left(D_{-}(\zeta)\right)^{c}}$.

We begin with the first case, which is illustrated in Fig. 1.

Let us denote the slope of $\zeta$ by $\alpha$. Since we assumed that $\xi$ is a minimizer, then we deduce from Proposition 1 that $\xi \in C^{1,1}([a, b])$. For a sufficiently small $\eta>0$, the line

$$
\ell(x)=\alpha\left(x-x_{0}\right)+\xi\left(x_{0}\right)-\eta
$$

intersects $\xi$ in $\left(x_{0}-\delta, x_{0}+\delta\right)$. Thus, function $h(x):=\min \{\ell(x), \xi(x)\}$ satisfies

$$
\zeta(x) \leqq h(x) \leqq \xi(x), \quad h\left(x_{0} \pm \delta\right)=\xi\left(x_{0} \pm \delta\right) .
$$

We will see that

$$
\int_{x_{0}-\delta}^{x_{0}+\delta}\left|h^{\prime}(x)\right|^{2} \mathrm{~d} x<\int_{x_{0}-\delta}^{x_{0}+\delta}\left|\xi^{\prime}(x)\right|^{2} \mathrm{~d} x
$$

which contradicts the minimality of $\xi$.

Indeed,

$$
\begin{aligned}
\int_{x_{0}-\delta}^{x_{0}+\delta}\left|h^{\prime}(x)\right|^{2} \mathrm{~d} x & =\int_{\{h=\xi\}}\left|h^{\prime}(x)\right|^{2} \mathrm{~d} x+\int_{\{h<\xi\}}\left|h^{\prime}(x)\right|^{2} \mathrm{~d} x \\
& =\int_{\{h=\xi\}}\left|\xi^{\prime}(x)\right|^{2} \mathrm{~d} x+\sum_{\mathcal{J}} \int_{\mathcal{J}} \alpha^{2} \mathrm{~d} x,
\end{aligned}
$$

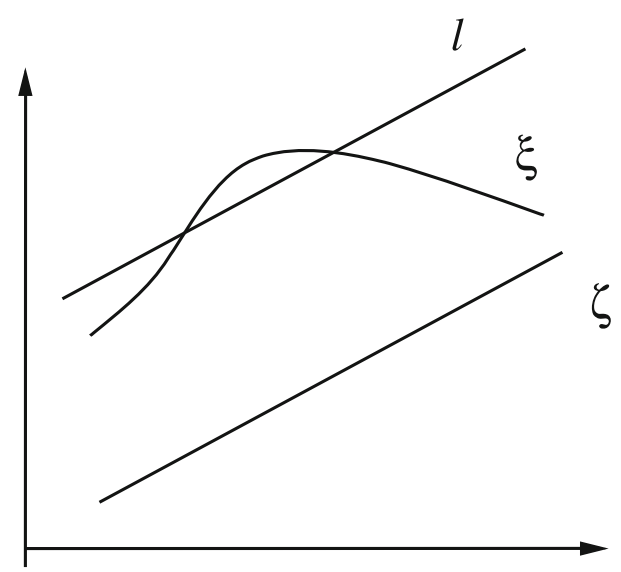

Fig. 1. The slope of $\zeta_{I}$ and $\ell$ is the same 
where $\mathcal{J}$ 's are the connected components of the set $\{h<\xi\}$. We notice that $h=\xi$ on $\partial \mathcal{J}$. Since the linear function minimizes the functional

$$
\int_{\mathcal{J}}\left|u^{\prime}(x)\right|^{2} \mathrm{~d} x
$$

among $H^{1}$-functions with given Dirichlet data, we conclude that

$$
\int_{\mathcal{J}} \alpha^{2} \mathrm{~d} x<\int_{\mathcal{J}}\left|\xi^{\prime}(x)\right|^{2} \mathrm{~d} x .
$$

Hence, 2.7 follows, and as a result the Lemma holds in case (i).

Let us consider case (ii). If it occurs, then $\zeta(x)=\psi(x)$ for $x \in\left(x_{0}-\delta, x_{0}+\delta\right)$. But first of all, $\xi^{\prime}\left(x_{0}\right)=\zeta^{\prime}\left(x_{0}\right)=: \alpha$ and we define $\ell$ in a similar way as in 2.6. Since $x_{0}$ is a local maximum, then $\xi^{\prime}(x) \geqq \zeta^{\prime}(x)$ for $x \in\left(x_{0}-\delta, x_{0}\right), \xi^{\prime}(x) \leqq \zeta^{\prime}(x)$ for $x \in\left(x_{0}, x_{0}+\delta\right)$. Thus, for a sufficiently small $\eta>0$ the line $\ell$ intersects $\xi$. From now on, we proceed as in the case (i) to show the impossibility of the local maximum.

The last case, (iii), uses the same kind of argument, as soon as we realize that the solution to the minimization problem with an obstacle meets the obstacle tangentially. This follows from the convexity-concavity condition in Proposition 1. The details are left to the interested Reader. This finishes the proof of the Lemma.

We may now turn our attention to the proof of Theorem 1

Proof. It suffices to prove

(a) $\Lambda_{++}\left(x, I_{1}\right) \leqq \Lambda_{++}\left(x, I_{2}\right), \Lambda_{--}\left(x, I_{2}\right) \leqq \Lambda_{--}\left(x, I_{1}\right)$ for $x \in I_{2} \subset I_{1}$,

(b) $\quad \Lambda_{-+}\left(x, I_{1}\right) \leqq \Lambda_{++}\left(x, I_{1}\right), \Lambda_{--}\left(x, I_{1}\right) \leqq \Lambda_{-+}\left(x, I_{1}\right)$ and $\Lambda_{+-}\left(x, I_{1}\right) \leqq \Lambda_{++}\left(x, I_{1}\right), \Lambda_{--}\left(x, I_{1}\right) \leqq \Lambda_{+-}\left(x, I_{1}\right)$ for $x \in I_{1}$.

We begin with the proof of (a). Since the argument is symmetric, it is sufficient to prove the first inequality. We may assume that one of the end points of $I_{1}$ and $I_{2}$ is the same. By symmetry, it suffices to prove that

$$
\Lambda_{++}\left(x, I_{1}\right) \leqq \Lambda_{++}\left(x, I_{2}\right), \quad x \in I_{2}
$$

with $I_{1}=(a, c), I_{2}=(a, b)$ for $c(\geqq b)$. Let us set $\xi:=\xi_{++, I_{2}}$, where $\xi_{++, I_{2}}^{\prime}=$ $\Lambda_{++}\left(x, I_{2}\right)$ and $\zeta:=\xi_{++, I_{1}}$, where $\xi_{++, I_{1}}^{\prime}=\Lambda_{++}\left(x, I_{1}\right)$. Due to the definition of $J_{\chi_{l} \chi_{r}, I}^{Z}$ and $K_{\chi_{l} \chi_{r}}^{Z}$ we conclude that

$$
\xi(a)=\zeta(a), \quad \xi(b) \geqq \zeta(b) .
$$

However, when $\xi(b)=\zeta(b)$, then there is nothing to prove, so we assume that $\xi(b)>\zeta(b)$. Thus, we may apply the Elementary Lemma, to deduce that $\xi^{\prime} \geqq \zeta^{\prime}$ in $I_{2}$, that is, 2.8 holds.

We next prove (b). By symmetry it suffices to show one of four inequalities. We shall prove that

$$
\Lambda_{--}\left(x, I_{1}\right) \leqq \Lambda_{-+}\left(x, I_{1}\right)
$$


Let $\zeta=\xi_{--, I_{1}}$ be the minimizer such that $\zeta^{\prime}=\Lambda_{--}\left(x, I_{1}\right)$ and let $\left.\xi=\xi_{-+, I_{2}}\right)$ be the minimizer such that $\xi^{\prime}=\Lambda_{-+}\left(x, I_{2}\right)$. By the structure of minimization problems, we see that $\xi(a)=\zeta(a)$ and $\xi(b) \geqq \zeta(b)$. We may directly apply the Elementary Lemma, to deduce that 2.9 holds.

\subsection{Stability of Curvature like Quantity}

Our goal in this section is to show that the curvature like quantity $\Lambda_{\chi_{l} \chi_{r}}(x, I)$ defined by (2.5) is 'continuous' with respect to the change of the interval $I$. The stability result for $\Lambda$ of the convex obstacle problem with respect to $Z$ is essentially known in the literature for example [35, p.156, Chapter 5, Theorem 4.5 and Remark 4.6]. However, we give a proof for the reader's convenience since the situation is slightly different.

We recall several stability properties of $J_{\chi_{l} \chi_{r}}$. Let $\left\{Z^{k}\right\}_{k=1}^{\infty}$ be a sequence of real-valued $C^{2}$ (or $C^{1,1}$ ) functions in $\bar{I}$, where $I=(a, b)$. In this subsection we fix $\chi_{l} \chi_{r}$, as a result we often suppress its dependence and simply write $J_{\chi_{l} \chi_{r}}^{Z}$ for $J$ and $J^{k}$ instead of $J_{\chi_{l} \chi_{r}}^{Z^{k}}$.

Proposition 2 (Lower semicontinuity). Assume that $Z^{k}$ uniformly converges to $Z$ as $k \rightarrow \infty$, that is, $Z^{k} \rightarrow Z$ in $C(\bar{I})$. Assume that $\xi_{k}$ weakly converges to $\xi$ in $L^{2}(I)$ as $k \rightarrow \infty$. Then $J(\xi) \leqq \liminf _{k \rightarrow \infty} J^{k}\left(\xi_{k}\right)$.

Proof. We may assume that $\xi_{k} \in K^{Z^{k}}$. Since $\xi^{k}-Z^{k}$ converges to $\xi-Z$ weakly in $L^{2}(I)$ and the sign is conserved through the weak limit, then we observe that $\xi \in K^{Z}$. The desired conclusion now follows from the lower semicontinuity of $H^{1}$-norm with respect to $L^{2}$-weak convergence.

Proposition 3 (Approximability). Assume that $Z^{k}$ converges to $Z$, with its first derivative, uniformly in $\bar{I}$ as $k \rightarrow \infty$, that is, $Z^{k} \rightarrow Z$ in $C^{1}(\bar{I})$. Then for each $\xi \in L^{2}(I)$, there is a sequence $\xi_{k} \rightarrow \xi$ in $L^{2}(I)$ such that $J(\xi)=\lim _{k \rightarrow \infty} J^{k}\left(\xi_{k}\right)$.

Proof. We may assume that $\xi \in K^{Z}$ since otherwise $\xi \notin K^{Z^{k}}$ for sufficiently large $k$. We set $\xi_{k}=\xi-Z+Z^{k}$ and observe that $\xi_{k}$ is in $K^{Z^{k}}$ by (2.3) and (2.4). Since $Z^{k} \rightarrow Z,\left(Z^{k}\right)^{\prime} \rightarrow Z^{\prime}$ uniformly in $\bar{I}$ as $k \rightarrow \infty$, the convergence $J\left(\xi_{k}\right) \rightarrow J(\xi)$ and $\xi_{k} \rightarrow \xi($ as $k \rightarrow \infty)$ in $L^{2}(I)$ is easily verified.

These two above Propositions say that $J^{k}$ converges to $J$ in the sense of Mosco, that is, both strong and weak $\Gamma^{-}$limits of $J^{k}$ equal $J$. Thus we easily obtain the convergence of minimizers.

Proposition 4 (Convergence of minimizers). Assume that $Z^{k} \rightarrow Z$ with its first derivative uniformly on $\bar{I}$, that is $Z^{k} \rightarrow Z$ in $C^{1}(\bar{I})$. Let $\xi_{\chi_{l} \chi_{r}}^{k}$ be the minimizer of $J_{\chi_{l} \chi_{r}}^{k}$. Then $\xi_{\chi_{l} \chi_{r}}^{k}$ converges to $\xi_{\chi_{l} \chi_{r}}$ in $L^{2}(I)$ which is the minimizer of $J_{\chi_{l} \chi_{r}}$.

Proof. We deduce from [33, Theorem 7.1]

$$
\operatorname{Lip}\left(\xi_{\chi_{l} \chi_{r}}^{k}\right) \leqq \max _{x \in \bar{I}}\left|Z_{x}^{k}(x)\right| \leqq \max _{k \in \mathbb{N}}\left\|Z^{k}\right\|_{C^{1}}<\infty
$$


This implies that $\left\{\min J^{k}\right\}_{k=1}^{\infty}$ is bounded. Since $H^{1}(I)$ is compactly embedded in $L^{2}(I)$, then upon extracting a subsequence $\xi_{\chi_{l} \chi_{r}}^{k}$ (not relabeled) converges to an element $\zeta \in L^{2}(I)$ as $k \rightarrow \infty$. By Proposition 2, we observe that

$$
J(\zeta) \leqq \liminf _{k \rightarrow \infty} \min J^{k}
$$

For any $\xi \in L^{2}(I)$, due to Proposition 3, there is always a sequence $\xi_{k} \rightarrow \xi$ in $L^{2}(I)$ such that $J^{k}\left(\xi_{k}\right) \rightarrow J(\xi)$ as $k \rightarrow \infty$. Thus

$$
J(\xi) \geqq \liminf _{k \rightarrow \infty} \min J^{k} .
$$

Therefore, $J(\zeta) \leqq J(\xi)$ so $\zeta$ must be the unique minimizer of $J$. Thus $\xi_{\chi_{l} \chi_{r}}^{k}$ converges $\xi_{\chi_{l} \chi_{r}}$ without taking a subsequence.

We define $\Lambda_{\chi_{l} \chi_{r}}^{k}(x, I)$ by (2.5) where $Z$ is replaced by $Z^{k}$. We simply write $\Lambda_{\chi_{l} \chi_{r}}^{k}$ in place of $\Lambda_{\chi_{l} \chi_{r}}^{k}(x, I)$ and $\Lambda_{\chi_{l} \chi_{r}}$ instead of $\Lambda_{\chi_{l} \chi_{r}}^{Z^{\prime}}(x, I)$ in the next Theorem.

Theorem 2 (Continuity with respect to $Z^{\prime}$ ). Assume that

$$
\sup _{k \geqq 1} \sup _{x \in I}\left|(\mathrm{~d} / \mathrm{d} x)^{2} Z^{k}(x)\right|<\infty \text { and }\left(Z^{k}\right)^{\prime} \rightarrow Z^{\prime} \text { in } C(\bar{I}) \text {. }
$$

Then $\Lambda_{\chi_{l} \chi_{r}}^{k} \rightarrow \Lambda_{\chi_{l} \chi_{r}}$ in $C(\bar{I})$ as $k \rightarrow \infty$.

Proof. We may assume that $Z^{k} \rightarrow Z$ in $C^{1}(\bar{I})$ by adding a constant to fix a value at some point of $I$, for example $Z^{k}((a+b) / 2)=0, Z((a+b) / 2)=0$. By Proposition 4 we observe that $\xi_{\chi_{l} \chi_{r}}^{k} \rightarrow \xi_{\chi_{l} \chi_{r}}$ in $L^{2}(I)$. By Proposition 1, our assumption on the bound of the second derivative of $Z^{k}$ implies that $\left|(\mathrm{d} / \mathrm{d} x)^{2} \xi_{\chi_{l} x_{r}}^{k}\right|$ is bounded by (2.4). Thus $\xi_{\chi_{l} \chi_{r}}^{k} \rightarrow \xi_{\chi_{l} \chi_{r}}$ in $C^{1}(\bar{I})$ so $\Lambda_{\chi_{l} \chi_{r}}^{k} \rightarrow \Lambda_{\chi_{l} \chi_{r}}$ in $C(\bar{I})$.

We are now in position to state the continuity of $\Lambda_{\chi_{l} \chi_{r}}$ with respect to $I$. This notion will be explained below.

Theorem 3. (i) Let $Z$ be a $C^{2}$ (or locally $C^{1,1}$ ) function on $\mathbf{R}$. Then $\Lambda_{\chi_{l} \chi_{r}}^{Z^{\prime}}(x, I)$ is continuous with respect to $I$.

(ii) Assume furthermore that $\left|Z^{\prime \prime}(x)\right|$ is bounded in $\mathbf{R}$. Then for each $r>0$

$$
\lim _{\mu \rightarrow 0} \sup _{0<b-a<r} \sup _{a<x<b}\left|\Lambda_{\chi_{l} \chi_{r}}^{Z^{\prime}}(x,(a, b))-\Lambda_{\chi_{l} \chi_{r}}^{Z^{\prime}}(x-\mu,(a-\mu, b-\mu))\right|=0 .
$$

(The convergence is uniform in $Z^{\prime}$ for $Z$ such that $\left|Z^{\prime \prime}\right| \leqq M_{0}$ for a given constant $M_{0}>0$ ).

We have to clarify the continuity with respect to $I$. For two bounded intervals $I=(a, b)$ and $J=(c, d)$ there is a unique affine map $A: x \mapsto y=\alpha x+\beta$ (dilation and translation) with $\alpha>0$ such that $A(I)=J$. Assume that an open interval $I^{k}$ converges to $I$ as $k \rightarrow \infty$, that is, the end points $a_{k}, b_{k}$ of $I_{k}=\left(a_{k}, b_{k}\right)$ tend to $a$ and $b$, respectively. Let $F$ be a mapping: $I \mapsto F(I) \in C(\bar{I})$. We say that $F$ is continuous with respect to $I$ if $F\left(I_{k}\right) \circ A_{k}$ converges to $F(I)$ in $C(I)$, as $k \rightarrow \infty$ for any $I^{k} \rightarrow I$, where $A^{k}$ is the affine map which maps $I$ to $I^{k}$. 
Proof. These assertions easily follow from Theorem 2, once we compare $\Lambda_{\chi_{l} \chi_{r}}^{Z^{\prime}}(x, I)$ with $\Lambda_{\chi_{l} \chi_{r}}^{Z^{\prime}}\left(A^{k}(x), I_{k}\right)$, both defined on $I$, here $A^{k}$ is the affine transformation mapping $I$ to $I^{k}$, when $I^{k} \rightarrow I$ (in the assertion (ii) this affine map is just a translation).

\subsection{Nonlocal Curvature with a Nonuniform Driving Force Term}

In order to define the nonlocal curvature $\Lambda_{W}^{\sigma}(u)$, formally given by (2.1), we recall basic assumptions on $W$ as in [17] and a class of function $u$, so that $\Lambda_{W}^{\sigma}(u)$ is well-defined.

(W) Let $W$ be a convex function on $\mathbf{R}$ with values in $\mathbf{R}$. Assume that $W$ is of class $C^{2}$ outside a closed discrete set $P$ and that $W^{\prime \prime}$ is bounded in any compact set except all points in $P$.

We shall always assume (W) in this paper. By definition, the set $P$ is either a finite set or a countable set having no accumulation points in $\mathbf{R}$. If $P$ is nonempty, $P$ is of form $\left\{p_{j}\right\}_{j=1}^{m},\left\{p_{j}\right\}_{j=-\infty}^{\infty},\left\{r_{j}\right\}_{j=-\infty}^{-1}$ or $\left\{p_{j}\right\}_{j=1}^{\infty}$ with $\lim _{j \rightarrow \infty} p_{j}=$ $\infty, \lim _{j \rightarrow-\infty} r_{j}=-\infty$, where the $p_{j}$ 's and $r_{j}$ 's are arranged in strictly increasing sequences $p_{j}<p_{j+1}, r_{j}<r_{j+1}$ and $m$ is a positive integer.

We recall a notion of a faceted function. Let $\Omega$ be an open interval. A function $f$ in $C(\Omega)$ is called faceted at $x_{0}$ with slope $p$ on $\Omega$ (or p-faceted at $x_{0}$ ) if there is a closed nontrivial finite interval $I(\subset \Omega)$ containing $x_{0}$ such that $f$ agrees with an affine function

$$
\ell_{p}(x)=p\left(x-x_{0}\right)+f\left(x_{0}\right) \text { in } I
$$

and $f(x) \neq \ell_{p}(x)$ for all $x \in J \backslash I$ with some neighborhood $J(\subset \Omega)$ of $I$. The interval $I$ is called a faceted region of $f$ containing $x_{0}$ and is denoted by $R\left(f, x_{0}\right)$. A function $f$ is called $P$-faceted at $x_{0}$ if it is $p$-faceted at $x_{0}$ for some $p \in P$.

We introduce the left transition number $\chi_{l}=\chi_{l}\left(f, x_{0}\right)$ and the right transition number $\chi_{r}=\chi_{r}\left(f, x_{0}\right)$ by

$$
\begin{aligned}
& \chi_{l}=\left\{\begin{array}{lll}
+1 \text { if } f \geqq \ell_{p_{i}} & \text { in } & \left\{x \in J \mid x \leqq x_{0}\right\} \\
-1 \text { if } f \leqq \ell_{p_{i}} & \text { in } & \left\{x \in J \mid x \leqq x_{0}\right\}
\end{array}\right. \\
& \chi_{r}=\left\{\begin{array}{lll}
+1 \text { if } f \geqq \ell_{p_{i}} & \text { in } & \left\{x \in J \mid x \geqq x_{0}\right\} \\
-1 \text { if } f \leqq \ell_{p_{i}} & \text { in } & \left\{x \in J \mid x \geqq x_{0}\right\}
\end{array}\right.
\end{aligned}
$$

if $f$ is $p_{i}$-faceted at $x_{0}$. The quantity $\chi=\left(\chi_{l}+\chi_{r}\right) / 2$ is called the transition number describing the sign of $\Lambda_{W}^{\sigma}$ when $\sigma \equiv 0$.

Definition 2. We assume that $\sigma$ is a real-valued Lipschitz function on an open interval $\Omega$ and $Z$ is its primitive, moreover, (W) holds. We assume that $f \in C(\Omega) p_{i}$ faceted at $x_{0} \in \Omega$ with $p_{i} \in P$. Then we define the nonlocal curvature $\Lambda_{W}^{\sigma}$ by

$$
\Lambda_{W}^{\sigma}(f)\left(x_{0}\right)=\Lambda_{\chi_{l} \chi_{r}}^{Z^{\prime}}(x, I)
$$


the right hand side is defined by (2.5) with $\Delta=W^{\prime}\left(p_{i}+0\right)-W^{\prime}\left(p_{i}-0\right)$ and $I$ is the faceted region $R\left(f, x_{0}\right)$. If $f$ is twice differentiable at $x_{0}$ and $f^{\prime}\left(x_{0}\right) \notin P$, we set, as expected,

$$
\Lambda_{W}^{\sigma}(f)\left(x_{0}\right)=W^{\prime \prime}\left(f^{\prime}\left(x_{0}\right)\right) f^{\prime \prime}\left(x_{0}\right)+\sigma\left(x_{0}\right) .
$$

Remark 1. If $\sigma$ is a constant, so that $Z$ is an affine function, the minimizer $\xi_{\chi_{l} \chi_{r}}$ of $J_{\chi_{l} \chi_{r}}^{Z}$ is always a straight line function (cf. Corollary 1 for the case $\chi=1$ or -1 ). Thus, it is easy to observe that

$$
\Lambda_{W}^{\sigma}(f)\left(x_{0}\right)=\chi \Delta / L\left(f, x_{0}\right)+\sigma\left(x_{0}\right)
$$

when $f$ is $p_{i}$-faceted at $x_{0}$, where $L\left(f, x_{0}\right)$ is the length of the faceted region $R\left(f, x_{0}\right)$. In particular, our new quantity agrees with the weighted curvature $\Lambda_{W}\left(f, x_{0}\right)$, defined in [17] when $\sigma \equiv 0$. Like $\Lambda_{W}\left(f, x_{0}\right)$, the quantity $\Lambda_{W}^{\sigma}$ depends on $W$ only through its second distributional derivative.

We conclude this section by rewriting the Comparison Principle and Continuity with respect to translation in terms of $\Lambda_{W}^{\sigma}$. Let $C_{P}^{2}(\Omega)$ be the set of $f \in C^{2}(\Omega)$ such that $f$ is $P$-faceted at $x_{0}$ whenever $f^{\prime}\left(x_{0}\right) \in P$. For such a class of function the nonlocal curvature $\Lambda_{W}^{\sigma}(f)(x)$ is well-defined for all $x \in \Omega$ provided that $\sigma$ is locally Lipschitz. The next two results are immediate consequences of Theorem 1 and Theorem 3, respectively.

Theorem 4 (Comparison). Assume condition (W) and that $\sigma$ is locally Lipschitz and in addition $f, g \in C_{P}^{2}(\Omega)$ and $x_{0} \in \Omega$. If $\max _{\Omega}(f-g)=(f-g)\left(x_{0}\right)$, then $\Lambda_{W}^{\sigma}(f)\left(x_{0}\right) \leqq \Lambda_{W}^{\sigma}(g)\left(x_{0}\right)$.

Theorem 5 (Continuity). Let us suppose that the hypotheses of Theorem 4 concerning $W$ and $\sigma$ hold. We assume that $f \in C(\Omega)$ is $p_{i}$-faceted at $x_{0}-\eta$ and $g$ be $p_{i}$-faceted at $x_{0}-\eta$ and $p_{i} \in P$. Assume moreover, $R\left(f, x_{0}\right)-\eta=R\left(g, x_{0}-\eta\right)$. Then

$$
\Lambda_{W}^{\sigma}(g)\left(x_{0}-\eta\right) \rightarrow \Lambda_{W}^{\sigma}(f)\left(x_{0}\right) \text { as }|\eta| \rightarrow 0 .
$$

\section{Definitions of Generalized Solutions}

The goal of this section is to define generalized solutions (in the viscosity sense) for evolution equations of the form

$$
u_{t}+F\left(t, u_{x}, \Lambda_{W}^{\sigma}(u)\right)=0
$$

when $W$ is a singular interfacial energy. Such a notion is given when $\sigma \equiv 0$ in [17]. Our definition will be a natural extension to the case when $\sigma \not \equiv 0$. In this section, we shall also give several equivalent definitions for later use. 


\subsection{Admissible Functions and Definitions}

We first recall a natural class of test function. Let us set $Q=(0, T) \times \Omega$, where $\Omega$ is an open interval and $T>0$. Let $A_{P}(Q)$ be the set of all admissible functions $\psi$ on $Q$ in the sense of [17] that is, $\psi$ is of the form

$$
\psi(x, t)=f(x)+g(t), f \in C_{P}^{2}(\Omega), g \in C^{1}(0, T) .
$$

For our equation, we often assume that

(F1) $F$ is continuous in $[0, T] \times \mathbf{R} \times \mathbf{R}$ with values in $\mathbf{R}$,

(F2) (Monotonicity) $F(t, p, X) \leqq F(t, p, Y)$ for $X \geqq Y, t \in[0, T], p \in \mathbf{R}$,

(FL) (Lipschitz continuity.) There is a constant $C=\bar{C}_{F, T}$ such that

$$
|F(t, p, X)-F(t, p, Y)| \leqq C(1+|p|)|X-Y| \text { for all } t \in[0, T], p, X, Y \in \mathbf{R} \text {. }
$$

(FT) (Uniform continuity in curvature and time.) For each $K$ the function $F(t, p, X)$ is uniformly continuous in $[0, T] \times[-K, K] \times \mathbf{R}$.

The third assumption is rather standard when $W \equiv 0$ and $\sigma$ is Lipschitz so that $\Lambda_{W}^{\sigma}(u)=\sigma$. A typical example of (3.1) satisfying (F1), (F2), (FL) and (FT) is of the form

$$
u_{t}-a\left(u_{x}\right) \Lambda_{W}^{\sigma}(u)-C(t)=0
$$

where

$$
F(t, p, X)=-a(t, p) X-C(t) ;
$$

here, $a \in C(\mathbf{R})$ satisfies $0 \leqq a(p) \leqq C(|p|+1)$ for all $p \in \mathbf{R}, C \in C[0, T]$. If $a(p)=\left(1+p^{2}\right)^{1 / 2}$ and $C \equiv 0$, then (3.2) says that the normal velocity $V$ of the graph of $u$ equals the nonlocal curvatures, that is, $V=\Lambda_{W}^{\sigma}$. The condition (FT) is redundant if $F$ is independent of $t$ since (FL) implies (FT).

The driving force term $\sigma$ may depend on $t$. Here is an assumption we often use. (S) The function $\sigma \in C([0, T] \times \bar{\Omega})$ is Lipschitz in space uniformly in time, that is there is a constant $L_{T}$ such that

$$
|\sigma(t, x)-\sigma(t, y)| \leqq L_{T}|x-y|
$$

for all $t \in[0, T], x, y \in \bar{\Omega}$.

We are now in the position to give a notion of a generalized solution in the viscosity sense.

Definition 3. Assume (W), (S), (F1), (F2). A real-valued function $u$ on $Q$ is a (viscosity) subsolution of (3.1) in $Q$ if the upper-semicontinuous envelope $u^{*}<\infty$ in $[0, T) \times \bar{\Omega}$ and

$$
\psi_{t}(\hat{t}, \hat{x})+F\left(\hat{t}, \psi_{x}(\hat{t}, \hat{x}), \Lambda_{W}^{\sigma(\hat{t},)}(\psi(\hat{t}))(\hat{x})\right) \leqq 0
$$

whenever $(\psi,(\hat{t}, \hat{x})) \in A_{P}(Q) \times Q$ fulfills

$$
\max _{Q}\left(u^{*}-\psi\right)=\left(u^{*}-\psi\right)(\hat{t}, \hat{x})
$$


Here, $\psi(\hat{t})$ is a function on $\Omega$ defined by $\psi(\hat{t})=\psi(\hat{t}, \cdot)$ and $u^{*}$ is defined by

$$
u^{*}(t, x)=\lim _{\varepsilon \downarrow 0} \sup \{u(s, y)|| s-t|<\varepsilon,| x-y \mid<\varepsilon,(s, y) \in Q\}
$$

for $(t, x) \in \bar{Q}$ and $u_{*}=\left(-u^{*}\right)$. A (viscosity) supersolution is defined by replacing $u^{*}(<\infty)$ by the lower-semicontinuous envelope $u_{*}(>-\infty)$, max by min in (3.4) and the inequality (3.3) by the opposite one. If $u$ is both a sub- and supersolution, it is called a viscosity solution or a generalized solution. Hereafter, we avoid using the word viscosity. Function $\psi$ satisfying (3.4) is called a test function of $u$ at $(\hat{t}, \hat{x})$.

The monotonicity, that is (F2), and the convexity, that is $(\mathrm{W})$, conditions show that the equation is at least degenerate parabolic. Thus, by comparison (Theorem 4 ), it is easy to see that $\psi \in A_{P}(Q)$ is a subsolution in $Q$ if (and only if) $\psi$ satisfies

$$
\psi_{t}(t, x)+F\left(t, \psi_{x}(t, x), \Lambda_{W}^{\sigma(t, \cdot)}(\psi(t))(x)\right) \leqq 0
$$

for all $(t, x) \in Q$.

\subsection{An Equivalent Definition}

To show the comparison principle for sub- and supersolutions, it is convenient to recall equivalent definitions. One of them is regarded as an infinitesimal version. Such a definition is given in [17] when $\sigma \equiv 0$. It is simplified by [21]. We give a definition which is a natural extension of the one in [21, Theorem 4.3].

We first recall upper time derivations on a faceted region. Let $\varphi$ be a function on $Q$ and $(\hat{t}, \hat{x}) \in Q$. Assume that $\varphi(\hat{t}, \cdot) \in C(\Omega)$ is $p$-faceted at $\hat{x} \in \Omega$ with $p \in P$. We define

$$
\begin{gathered}
\mathcal{T}_{P}^{+} \varphi(\hat{t}, \hat{x})=\{\tau \in \mathbf{R} \mid \text { there are a modulus } \\
\omega \text { and three positive numbers } \delta, \delta_{+}, \delta_{-} \text {such that } \\
\varphi(t, x)-\varphi(\hat{t}, \hat{x}) \leqq \tau(t-\hat{t})+p(x-\hat{x})+\omega(|\hat{t}-t|)|t-\hat{t}| \\
\text { for } \left.(t, x) \in(\hat{t}-\delta, \hat{t}+\delta) \times \tilde{N}^{-1}\left(\varphi(\hat{t}, \cdot), \hat{t} ; \delta_{+}, \delta_{-}\right)\right\},
\end{gathered}
$$

where $\tilde{N}^{-1}$ denotes a semineighborhood of $R(\varphi(\hat{t}, \cdot), \hat{x})$, defined in [17]; by a modulus $\omega$ we mean that $\omega:[0, \infty) \rightarrow[0, \infty)$ is nondecreasing, continuous with $\omega(0)=0$. For the reader's convenience, we recall the definition of $\tilde{N}^{-1}$. Let $f \in C(\Omega)$ be $p$-faceted at $x_{0} \in \Omega$ with $p \in P$. We set

$$
\begin{aligned}
& N_{\chi_{r}}\left(f, x_{0} ; \delta_{+}\right) \\
& \quad= \begin{cases}\left\{x \in \Omega \mid \sup R\left(f, x_{0}\right)<x \leqq \sup R\left(f, x_{0}\right)+\delta_{+}\right\} & \text {if } \chi_{r}\left(f, x_{0}\right)=-1, \\
\emptyset & \text { if } \chi_{r}\left(f, x_{0}\right)=1\end{cases} \\
& N_{\chi_{l}}\left(f, x_{0} ; \delta_{-}\right) \\
& = \begin{cases}\left\{x \in \Omega \mid \inf R\left(f, x_{0}\right)-\delta_{-} \leqq x<\inf R\left(f, x_{0}\right)\right\} & \text { if } \chi_{l}\left(f, x_{0}\right)=-1, \\
\emptyset & \text { if } \chi_{l}\left(f, x_{0}\right)=1\end{cases}
\end{aligned}
$$

and the set $\tilde{N}^{-1}$ is defined by (Fig. 2)

$$
\tilde{N}^{-1}\left(f, x_{0} ; \delta_{-}, \delta_{+}\right)=R\left(f, x_{0}\right) \cup N_{\chi_{r}}\left(f, x_{0} ; \delta_{+}\right) \cup N_{\chi_{l}}\left(f, x_{0} ; \delta_{-}\right) .
$$



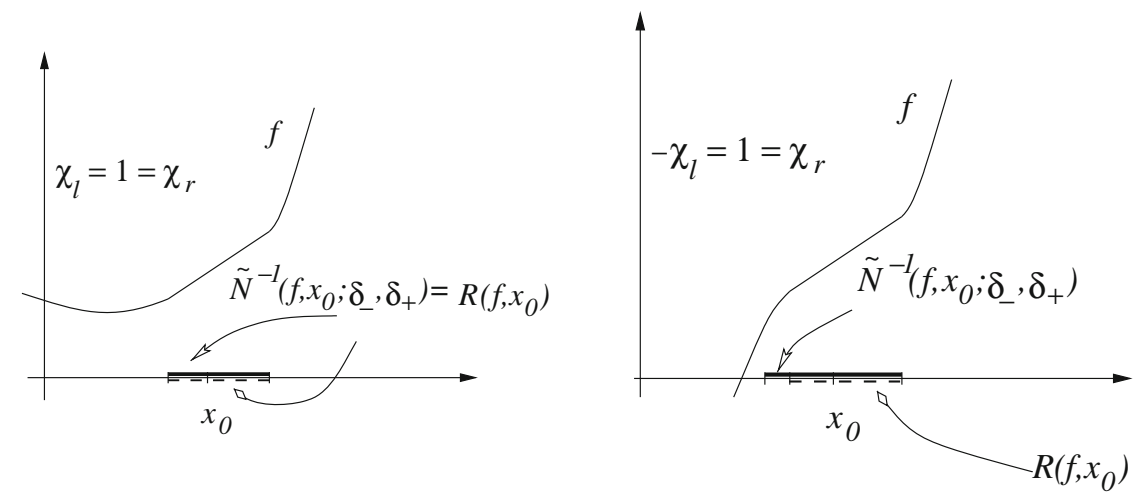

Fig. 2. Various possibilities of $\tilde{N}^{-1}\left(f, x_{0} ; \delta_{-}, \delta_{+}\right)$

The set $\tilde{N}^{+1}$ is defined by

$$
\tilde{N}^{+1}\left(f, x_{0} ; \delta_{-}, \delta_{+}\right)=\tilde{N}^{-1}\left(-f, x_{0} ; \delta_{-}, \delta_{+}\right) .
$$

An element of $\mathcal{T}_{P}^{+} \varphi(\hat{t}, \hat{x})$ is an upper time derivative at $(\hat{t}, \hat{x})$. The set of lower time derivatives is defined by

$$
\mathcal{T}_{P}^{-} \varphi(\hat{t}, \hat{x})=-\mathcal{T}_{-P}^{+}(-\varphi)(\hat{t}, \hat{x}) .
$$

We next recall a class of functions (not necessarily admissible) for which the upper time derivative is well-defined on a faceted region. The following definition is an improved one in [21], not the original one in [17]. In [21] $Q$ may not be noncylindrical but here, we consider a simple case $Q=(0, T) \times \Omega$.

Definition 4. Let $\varphi: \Omega \rightarrow \mathbf{R}$ be an upper-semicontinuous function. For $(\hat{t}, \hat{x}) \in Q$ assume that $\varphi(t, \cdot) \in C(\Omega)$ for $t$ near $\hat{t}$. We say that $\varphi$ is an (infinitesimally) admissible superfunction at $(\hat{t}, \hat{x})$ in $Q$ if one of the following three conditions holds.

(A) The function $\varphi(\hat{t}, \cdot)$ is $P$-faceted (in $\Omega$ ) at $\hat{x} \in$ int $R(\varphi(\hat{t}, \cdot), \hat{x})$. The set $\mathcal{T}_{P}^{+} \varphi(\hat{t}, \hat{x})$ is nonempty.

(B) There is $(\tau, p, X) \in \mathcal{P}^{+} \varphi(\hat{t}, \hat{x})$ with $p \notin P$, where $\mathcal{P}^{+}$denotes the set of parabolic semijets in $Q[11,17]$.

(C) The function $\varphi(\hat{t}, \cdot)$ is $P$-faceted at $\hat{x}$ but $\hat{x} \in \partial R(\varphi(\hat{t}, \cdot), \hat{x})$. There is an element $(\tau, p, 0) \in\left(\mathcal{P}^{+} \varphi(\hat{t}, \hat{x})\right)$ for some $\tau \in R$.

We say that $\varphi$ is an admissible subfunction at $(\hat{t}, \hat{x})$ in $Q$ if $\varphi$ is an admissible superfunction with $P$ replaced by $-P$. We implicitly assume that $R(\varphi(\hat{t}, \cdot), \hat{x})$ does not touch the boundary of $\Omega$. We are now in the position to give a definition of a subsolution in the infinitesimal sense.

Definition 5. Assume (W), (S), (F1), (F2). A real-valued function $u$ on $Q$ is a subsolution in the infinitesimal sense of (3.1) (in $Q$ ) if $u^{*}<\infty$ in $[0, T) \times \bar{\Omega}$ and the following conditions are fulfilled. For $(\hat{t}, \hat{x})$, let $\varphi$ be an admissible superfunction at $(\hat{t}, \hat{x})$ in $Q$ such that $\varphi$ is a test function of $u$ at $(\hat{t}, \hat{x})$, that is, (3.4) holds. Then 
(i) $\tau+F\left(\hat{t}, \varphi_{x}(\hat{t}, \hat{x}), \Lambda_{W}^{\sigma(\hat{t}, \cdot)}(\varphi(\hat{t}, \cdot))(\hat{x})\right) \leqq 0$ for all $\tau \in \mathcal{T}_{P}^{+} \varphi(\hat{t}, \hat{x})$ if (A) in Definition 4 holds;

(ii) $\tau+F\left(\hat{t}, p, W^{\prime \prime}(p) X+\sigma(\hat{t}, \hat{x})\right) \leqq 0$ for all $(\tau, p, X) \in \mathcal{P}^{+} \varphi(\hat{t}, \hat{x})$ if (B) in Definition 4 holds;

(iii) $\tau+F(\hat{t}, p, \sigma(\hat{t}, \hat{x})) \leqq 0$ for all $(\tau, p, 0) \in \mathcal{P}^{+} \varphi(\hat{t}, \hat{x})$ if $(\mathrm{C})$ in Definition 4 holds and

$$
\left(u^{*}-\varphi\right)(\hat{t}, x)<\max _{Q}\left(u^{*}-\varphi\right)
$$

for all $x \in R(\varphi(\hat{t}, \cdot), \hat{x}) \backslash\{\hat{x}\}$ near $\hat{x}$.

The definition of the supersolution in the infinitesimal sense is given by replacing $u^{*}(<\infty)$ by $u_{*}(>-\infty)$, max by min in (3.4), superfunction by subfunction, $\mathcal{T}_{P}^{+}$by $\mathcal{T}_{P}^{-}, \mathcal{P}^{+}$by $\mathcal{P}^{-}$and the inequalities in (i), (ii), (iii) by the opposite ones. It turns out that Definitions 3 and 5 are equivalent.

Theorem 6 (Equivalence). Assume $(W),(S),(F 1),(F 2)$. A real-valued function $u$ on $Q$ is a subsolution (resp. supersolution) of (3.1) in $Q$ if and only if $u$ is a subsolution (resp. supersolution) of (3.1) in $Q$ in the infinitesimal sense.

The proof essentially parallels that of [17, Theorem 6.9] and [21, Theorem 4.3]. In the proof of the 'only part', (iii) follows from the zero-curvature lemma [21, Lemma 4.2], with a trivial modification. We give a modified version of this lemma for reader's convenience. We do not repeat the tedious details of the proof of the 'only if' part. The proof of the 'if' part is easier and written in the proof of [21, Theorem 4.3]; of course we need trivial modifications, for example $\Lambda_{W}(\psi(\hat{t}, \cdot), \hat{x})<0$ should be replaced by $\chi(\psi(\hat{t}, \cdot), \hat{x})<0$.

Lemma 2 (Zero curvature). Let $u$ be a subsolution of (3.1) in $Q$. Assume that $\varphi \in A_{P}(Q)$ and that

$$
\max _{Q}\left(u^{*}-\varphi\right)=\left(u^{*}-\varphi\right)(\hat{t}, \hat{x})
$$

for $(\hat{t}, \hat{x}) \in Q$. If $\hat{x}$ is an end point of a faceted region $R(\varphi(\hat{t}, \cdot), \hat{x})$ with $\varphi_{x}(\hat{t}, \hat{x}) \in$ $P$ and $\left(u^{*}-\varphi\right)(\hat{t}, x)<\left(u^{*}-\varphi\right)(\hat{t}, \hat{x})$ for all $x \in R(\varphi(\hat{t}, \cdot), \hat{x})$ near $\hat{x}$, then

$$
\varphi_{t}(\hat{t}, \hat{x})+F\left(\hat{t}, \varphi_{x}(\hat{t}, \hat{x}), \sigma(\hat{t}, \hat{x})\right) \leqq 0 .
$$

\section{Comparison Principle}

We state our main comparison result for equation (3.1).

Theorem 7. (Comparison) Assume that condition $(W),(S),(F 1),(F 2),(F L)$ and $(F T)$ hold. Assume that $P$ is a finite set. Let $u$ and $v$ be respectively sub-and supersolutions of (3.1) in $Q=(0, T) \times \Omega$, where $\Omega$ is a bounded open interval. If $u^{*} \leqq v_{*}$ on the parabolic boundary $\partial_{p} Q(=[0, T) \times \partial \Omega \cup\{0\} \times \bar{\Omega})$ of $Q$, then $u^{*} \leqq v_{*}$ in $Q$. 
The proof will be given in the remaining part of this section. The basic strategy is in finding suitable test functions of $u$ and $v$ to obtain a contradiction after having assumed that the conclusion $u^{*} \leqq v_{*}$ had been false. This basic strategy is the same as in [17]. However, the nonlocal curvature may depend on $x$ even if $x$ is in a faceted region. So one should be careful on this issue. This is a new aspect of the problem. On the other hand since the infinitesimal version of definitions of sub- and supersolutions are simplified in comparison with [17], we need not avoid handling the case where functions take a maximum value at the end points of faceted regions. In fact, it is mentioned in [21] that the proof of [17] is simplified.

\subsection{Doubling Variables}

As usual, we double the variables. For $z=(t, x), z^{\prime}=(s, y) \in Q$, we set

$$
w\left(z, z^{\prime}\right)=u(z)-v\left(z^{\prime}\right) .
$$

We take a barrier function which is different from the one in [17]. Let $B \in C_{P}^{2}(\mathbf{R})$ be a function such that $B$ is convex, $x B^{\prime}(x) \geqq 0$ for all $x \in \mathbf{R}$ with $B(0)=0$ and

$$
0<\underline{\lim }_{|x| \rightarrow \infty} B^{\prime}(x) / x, \varlimsup_{|x| \rightarrow \infty} B^{\prime}(x) / x<\infty .
$$

Moreover, the length of all faceted regions is the same. It is easy to find the derivative $B^{\prime}$ of such a function by modifying $y=x$, so that $B$ is obtained as its primitive. We consider its rescaled version: $B_{\varepsilon}(x)=\varepsilon B(x / \varepsilon)$ for $\varepsilon>0$. Clearly, $B_{\varepsilon} \in C_{P}^{2}(\mathbf{R})$ and satisfies the same properties as $B$ 's. We consider 'barrier functions' of the diagonal $z=z^{\prime}$ :

$$
\begin{aligned}
& \Psi\left(z, z^{\prime} ; \varepsilon, \delta, \gamma, \gamma^{\prime}\right)=B_{\varepsilon}(x-y)+S\left(t, s ; \delta, \gamma, \gamma^{\prime}\right) \\
& S\left(t, s, \delta, \gamma, \gamma^{\prime}\right)=(t-s)^{2} / \delta+\gamma /(T-t)+\gamma^{\prime} /(T-s)
\end{aligned}
$$

for positive parameters $\varepsilon, \delta, \gamma, \gamma^{\prime}$ (in [17] we use $|x-y-\zeta|^{2} / \varepsilon^{2}$ instead of $B_{\varepsilon}(x-$ $y$ ), where $\zeta$ is an extra shift parameter used to avoid the situation when a point that we are dealing with is an end point of faceted regions). We often write $\Psi\left(z, z^{\prime}\right)$ and $S(t, s)$ instead of showing the dependence on all positive parameters. As usual, we shall analyze the maximizers of

$$
\Phi\left(z, z^{\prime}\right)=w\left(z, z^{\prime}\right)-\Psi\left(z, z^{\prime}\right)
$$

\subsection{Choice of Parameters}

We shall choose $\varepsilon, \delta, \gamma, \gamma^{\prime}$ sufficiently small, as usual. The next statement for the behavior of the maximizer of $\Phi$ is rather standard in the process of doubling variables; see for example, [16], [17, Proposition 7.1], [26].

Proposition 5. Assume that $u$ and $-v$ are upper-semicontinuous in $[0, T) \times \bar{\Omega}$ with values in $\mathbf{R} \cup\{-\infty\}$ and $u=u^{*}, v=v_{*}$ including $\{T\} \times \bar{\Omega}$, where $\Omega$ is an open set in $\mathbf{R}$. Assume that $m_{0}=\sup _{z \in Q} w(z, z)>0$. 
(i) For each $m_{0}^{\prime}$, $\left(0<m_{0}^{\prime}<m_{0}\right)$, there are $\gamma_{0}, \gamma_{0}^{\prime}>0$ such that $\sup _{Q \times Q} \Phi>m_{0}^{\prime}$ for all $\varepsilon>0, \delta>0, \gamma_{0}>\gamma>0, \gamma_{0}^{\prime}>\gamma^{\prime}>0$.

(ii) (Behavior of a maximizer) Let $\left(\hat{z}, \hat{z}^{\prime}\right)=(\hat{t}, \hat{x}, \hat{s}, \hat{y})$ be a maximizer of $\Phi$ over $\bar{Q} \times \bar{Q}$. Then

$$
|\hat{t}-\hat{s}| \leqq M \delta^{1 / 2}, B_{\varepsilon}(\hat{x}-\hat{y}) \leqq M
$$

with $M=\sup _{\bar{Q} \times \bar{Q}} w$ for all $\varepsilon>0, \delta>0, \gamma_{0}>\gamma>0$ and $\gamma_{0}^{\prime}>\gamma^{\prime}>0$. Moreover, $|\hat{t}-\hat{s}|^{2} / \delta \rightarrow 0, B_{\varepsilon}(\hat{x}-\hat{y}) \rightarrow 0$ since $M \rightarrow m$ as $\varepsilon \rightarrow 0, \delta \rightarrow 0$.

(iii) (Effect of boundary condition) Assume that $u \leqq v$ on $\bar{\partial}_{p} Q\left(=\overline{\partial_{p} Q}\right)$ and that $\Omega$ is a bounded open interval. Then, there are $\varepsilon_{0}, \delta_{0}$ such that $\left(\hat{z}, \hat{z}^{\prime}\right)$ is an (interior) point of $Q \times Q$ for all $0<\varepsilon<\varepsilon_{0}, 0<\delta<\delta_{0}, 0<\gamma<\gamma_{0}, 0<\gamma^{\prime}<\gamma_{0}^{\prime}$.

Remark 2. Since $w$ is upper-semicontinuous, we may assume in (iii) that for each $\xi>0$

$$
w\left(z, z^{\prime}\right) \leqq \xi \text { for }\left(z, z^{\prime}\right) \in \bar{\partial}_{p} Q \times \bar{Q} \cup \bar{Q} \times \bar{\partial}_{p} Q
$$

satisfying $B_{\varepsilon}(x-y)<M,|t-s|^{2} / \delta<M$ with $z=(t, s), z^{\prime}=(s, y)$.

In the sequel, we assume that $m_{0}>0$ with $\xi=\frac{1}{4} m_{0}, m_{0}^{\prime}=m_{0}-\xi / 2$ and we fix $\varepsilon_{0}, \delta_{0}, \gamma_{0}, \gamma_{0}^{\prime}$ so that all properties (i)-(iii) and those in Remark 2 hold.

\subsection{Maximizers in a Faceted Region of Test Functions}

We shall consider three cases depending on the location of maximizers $\left(\hat{z}, \hat{z}^{\prime}\right)=$ $(\hat{t}, \hat{x}, \hat{s}, \hat{y})$ of $\Phi$ over $\bar{Q} \times \bar{Q}$.

Case A: $\hat{p}=B_{\varepsilon}^{\prime}(\hat{x}-\hat{y}) \in P$ and $\hat{x}-\hat{y} \in$ int $R\left(B_{\varepsilon}, \hat{x}-\hat{y}\right)$.

Case $\mathrm{B}: \hat{p}=B_{\varepsilon}^{\prime}(\hat{x}-\hat{y}) \notin P$.

Case $\mathrm{C}: \hat{p}=B_{\varepsilon}^{\prime}(\hat{x}-\hat{y}) \in P$ and $\hat{x}-\hat{y} \in \partial R\left(B_{\varepsilon}, \hat{x}-\hat{y}\right)$.

Proposition 6. Assume the conditions of Case A for $\left(\hat{z}, \hat{z}^{\prime}\right)=(\hat{t}, \hat{x}, \hat{s}, \hat{y}) \in Q \times Q$. Let $u_{0}$ and $v_{0}$ denote

$$
u_{0}(t, x)=u(t, x)-\hat{p} x, v_{0}(s, y)=v(s, y)-\hat{p} y
$$

with $\hat{p}=B^{\prime}(\hat{x}-\hat{y})$. Then $u_{0}(\hat{t}, \cdot),-v_{0}(\hat{s}, \cdot)$ take their local maxima at $\hat{x}$ and $\hat{y}$ respectively. Moreover,

$$
u_{0}(t, x)-v_{0}(s, y)-S(t, s) \leqq u_{0}(\hat{t}, \hat{x})-v_{0}(\hat{s}, \hat{y})-S(\hat{t}, \hat{s})
$$

for all $(x, y) \in \Sigma_{\kappa}, t, s, \in[0, T]$ for sufficiently small $\kappa>0$ where

$$
\Sigma_{\kappa}=\{(x, y) \in \bar{\Omega} \times \bar{\Omega}|| x-y-(\hat{x}-\hat{y}) \mid<\kappa\} .
$$

This follows from the definition since $B_{\varepsilon}$ is a $P$-faceted function (we do not even invoke Proposition 5). 
Proposition 7 (No touching of faceted region on the boundary). Assume the conditions of Case A for $\left(\hat{z}, \hat{z}^{\prime}\right)$ and choose parameters $\varepsilon_{0}, \delta_{0}, \gamma_{0}, \gamma_{0}^{\prime}$ as in Remark 2. Assume that $0<\varepsilon<\varepsilon_{0}, 0<\delta<\delta_{0}, 0<\gamma<\gamma_{0}, 0<\gamma^{\prime}<\gamma_{0}^{\prime}$. Let $\Omega$ denote $\Omega=(a, b)$. Then there is $x_{1} \in\left(\hat{x}, b_{1}\right)$ or $y_{1} \in\left(\hat{y}, b_{2}\right)$ such that

$$
u_{0}\left(\hat{t}, x_{1}\right)<u_{0}(\hat{t}, \hat{x}) \text { or } v_{0}\left(\hat{s}, y_{1}\right)>v_{0}(\hat{s}, \hat{y})
$$

with $\eta=\hat{x}-\hat{y}, b_{1}=\min (b, b+\eta), b_{2}=\min (b, b-\eta)$. The same assertion is valid if $\left(\hat{x}, b_{1}\right)$ and $\left(\hat{y}, b_{2}\right)$ are replaced by $(a, \hat{x})$ and $\left(a_{2}, \hat{y}\right)$ respectively, with $a_{1}=\max (a, a+\eta), a_{2}=\max (a, a-\eta)$.

For the proof, we invoke Remark 2 . The proof depends on the boundary condition (Proposition 5 (iii)) and it parallels that of [17, Proposition 7.10].

\subsection{Existence of Admissible Superfunctions}

Unfortunately, functions $u_{0}$ and $v_{0}$ may not be faceted at $\hat{x}$ and $\hat{y}$. We have to regularize them by taking sup-convolution with faceted functions. For $\rho>0$ let $\vartheta(x, \rho)$ denote

$$
\vartheta(x, \rho)= \begin{cases}(x-\rho)^{2} / \rho, & x>\rho, \\ 0 & |x| \leqq \rho \\ (x+\rho)^{2} / \rho & x<-\rho .\end{cases}
$$

We consider sup-convolutions of $u_{0}$ and $-v_{0}$ by $\vartheta$. For $\alpha>0$ let $u_{0}^{\alpha}$ be the supconvolution of $u_{0}$ in the $x$-direction, that is,

$$
u_{0}^{\alpha}(t, x)=\left(u_{0}(t, \cdot)\right)^{\alpha}=\sup \left\{u_{0}(t, \xi)-\vartheta(\xi-x, \alpha) ; \xi \in \mathbf{R}\right\}
$$

where we use the convention that $u_{0}=-\infty$ if $\xi \notin \Omega$. The inf-convolution of $v_{0}$ is defined by $v_{0 \rho}=-\left(-v_{0}\right)^{\beta}$ for $\beta>0$. Functions $u_{0}^{\alpha}, v_{0 \beta}$ are defined in $[0, T] \times \mathbf{R}$. Based on these regularizations and the maximum principle for faceted sub- and supersolutions, the desired admissible super- and subfunctions are constructed. The proof is essentially the same as in [17, Proposition 7.12-7.15]. Although it is highly nontrivial, we do not repeat the proof.

Theorem 8. Assume the condition of Case A and choose parameters $\varepsilon_{0}, \delta_{0}, \gamma_{0}, \gamma_{0}^{\prime}$, as in Remark 2. Let $0<\varepsilon<\varepsilon_{0}, 0<\delta<\delta_{0}, 0<\gamma<\gamma_{0}$ and $0<\gamma^{\prime}<\gamma_{0}^{\prime}$. Then, there exists an admissible superfunction $U$ at $(\hat{t}, \hat{x})$ in $Q$ and an admissible subfunction $V$ at $(\hat{s}, \hat{y})$ in $Q$ satisfying the following properties.

(i) $U$ and $V$ are test functions of $u$ and $v$ at $(\hat{t}, \hat{x})$ and $(\hat{s}, \hat{y})$ respectively. In fact,

$$
\max _{Q}(u-U)=(u-U)(\hat{t}, \hat{x})=0, \min _{Q}(v-V)=(v-V)(\hat{s}, \hat{y})=0 .
$$

(ii) $U(\hat{t}, \cdot)$ is $\hat{p}$-faceted at $\hat{x} \in \operatorname{int} R(U(\hat{t}, \cdot), \hat{x})$ and $\mathcal{T}_{P}^{+} U(\hat{t}, \hat{x}) \ni S_{t}(\hat{t}, \hat{s})$;

$V(\hat{s}, \cdot)$ is $\hat{p}$-faceted at $\hat{y} \in \operatorname{int} R(V(\hat{s}, \cdot), \hat{y})$ and $\mathcal{T}_{P}^{-} V(\hat{s}, \hat{y}) \ni S_{S}(\hat{t}, \hat{s})$.

(iii) $R((U(\hat{t}, \cdot), \hat{x})=R(V(\hat{s}, \cdot), \hat{y})+(\hat{x}-\hat{y})$. In particular, $L(U(\hat{t}, \cdot), \hat{x})=$ $L(V(\hat{s}, \cdot), \hat{y})$.

(iv) $\chi(U(\hat{t}, \cdot), \hat{x})+\chi(-V(\hat{s}, \cdot), \hat{y}) \leqq 0$. 
The function $u_{0}^{\alpha}+p_{0} x$ is essentially an admissible superfunction so we are tempted to set $U=u_{0}^{\alpha}+p_{0} x$. However, the faceted region may contain the boundary point of $\partial \Omega$. Since

$u_{0}^{\alpha}(t, x)-v_{0 \alpha}(s, y) \leqq u_{0}^{\alpha}(\hat{t}, \hat{x})-v_{0 \alpha}(\hat{s}, \hat{y})+\vartheta\left(x-y-\eta, \frac{\lambda_{0}}{2}\right)+S(t, s)-S(\hat{t}, \hat{s})$

on $([0, T] \times \mathbf{R})^{2}$ for sufficiently small $\alpha$ as observed in [17, Proposition 7.13], we are able to apply the maximum principle for faceted functions [17, Corollary 4.6] to construct $U$. The properties (ii)-(iv) are obtained by the comparison principle for $\Lambda_{W}^{\sigma}$ (Theorems 1,4).

\subsection{Proof of Comparison Theorem}

We are now in position to prove Theorem 7. Suppose that the conclusion were false. We may assume that $u$ and $v$ satisfy the assumptions of Proposition 5, by considering $u^{*}$ and $v_{*}$ on $\bar{Q}$. In particular, we may assume $m_{0}>0$. We shall fix $\varepsilon_{0}, \delta_{0}, \gamma_{0}, \gamma_{0}^{\prime}$, as in Remark 2 , and assume that $0<\varepsilon<\varepsilon_{0}, 0<\delta<\delta_{0}, 0<\gamma<$ $\gamma_{0}$ and $0<\gamma^{\prime}<\gamma_{0}^{\prime}$. Since $\bar{Q}$ is compact and $u$ and $-v$ are upper-semicontinuous, there is always a maximizer $\left(\hat{z}, \hat{z}^{\prime}\right)=(\hat{t}, \hat{x}, \hat{y}, \hat{s})$ of $\Phi$ over $\bar{Q} \times \bar{Q}$ and it is in $Q \times Q$ by the choice of parameters (Proposition 5 (iii) and Remark 2). We shall fix $\gamma$ and $\gamma^{\prime}$. We divide the situations into three cases.

Case I. For sufficiently small $\varepsilon, \delta(>0)$ say $\varepsilon<\varepsilon_{1}\left(<\varepsilon_{0}\right), \delta<\delta_{1}\left(<\delta_{0}\right)$, there is a maximizer $\left(\hat{z}, \hat{z}^{\prime}\right)$ such that Case A occurs (for $\hat{x}$ and $\hat{y}$ ).

Case II. There is a sequence $\varepsilon_{j} \rightarrow 0, \delta_{j} \rightarrow 0$, such that there is a maximizer $\left(\hat{z}, \hat{z}^{\prime}\right)$, such that Case B occurs.

Case III. There is a sequence $\varepsilon_{j} \rightarrow 0, \delta_{j} \rightarrow 0$ such that there is a maximizer $\left(\hat{z}, \hat{z}^{\prime}\right)$ such that Case $C$ occurs and there is no maximizer $\left(\hat{z}, \hat{z}^{\prime}\right)$ such that either Case A or Case B occurs.

In Case I, we invoke Theorem 8. Since $U$ is an admissible superfunction at $(\hat{t}, \hat{x})$ in $Q$ and since $u$ is a subsolution, by Definition 4 and Theorem 8 (i), (ii) we have

$$
S_{t}(\hat{t}, \hat{s})+F\left(\hat{t}, \hat{p}, \Lambda_{W}^{\sigma(\hat{t}, \cdot)}(U(\hat{t}, \cdot))(\hat{x})\right) \leqq 0 .
$$

Similarly,

$$
-S_{S}(\hat{t}, \hat{s})+F\left(\hat{s}, \hat{p}, \Lambda_{W}^{\sigma(\hat{s}, \cdot)}(V(\hat{s}, \cdot))(\hat{y})\right) \geqq 0 .
$$

By Theorem 8 (iv), we have

$$
\begin{gathered}
\Lambda_{W}^{\sigma(\hat{t}, \cdot)}(U(\hat{t}, \cdot))(\hat{x})=\Lambda_{\chi_{l}^{U} \chi_{r}^{U}}^{\sigma(\hat{x}, \cdot)}\left(\hat{x}, I_{U}\right) \leqq \Lambda_{\chi_{l}^{V} \chi_{r}^{V}}^{\sigma(\hat{x} \cdot)}\left(\hat{x}, I_{U}\right), \\
I_{U}=R(U(\hat{t}, \cdot), \hat{x})
\end{gathered}
$$

where $\chi_{l}^{U}$ and $\chi_{r}^{U}$ denote the transition numbers of $U(\hat{t}, \cdot)$ on $I_{U}$ and $\chi_{l}^{V}$ and $\chi_{r}^{V}$ denote the transition numbers of $V(\hat{s}, \cdot)$ on $I_{V}=R(V(\hat{s}, \cdot), \hat{y})$. Since we have 
assumed that $P$ is a finite set, there is $K$ such that $P \subset[-K, K]$. Thus, by (FT) and (F2), inequalities (4.1) and (4.3) yield

$$
S_{t}(\hat{t}, \hat{s})+F\left(\hat{s}, \hat{p}, \Lambda_{\chi_{l}^{V} \chi_{r}^{V}}^{\sigma(\hat{t}, \cdot)}\left(\hat{x}, I_{U}\right)\right)-\omega_{K}(\hat{t}-\hat{s}) \leqq 0
$$

with some modulus $\omega_{K}$. By definition, inequality (4.2) can be rewritten as

$$
-S_{S}(\hat{t}, \hat{s})+F\left(\hat{s}, \hat{p}, \Lambda_{\chi_{l}^{V} \chi_{r}^{V}}^{\sigma(\hat{y}, \cdot)}\left(\hat{y}, I_{V}\right)\right) \geqq 0 .
$$

Subtracting (4.6) from (4.5) yields

$$
\begin{aligned}
& \frac{\gamma}{(T-\hat{t})^{2}}+\frac{\gamma^{\prime}}{(T-\hat{s})^{2}}+F\left(\hat{s}, \hat{p}, \Lambda_{\chi_{l}^{V} \chi_{r}^{V}}^{\sigma(\hat{t}, \cdot)}\left(\hat{x}, I_{U}\right)\right)-F\left(\hat{s}, \hat{p}, \Lambda_{\chi_{l}^{V} \chi_{r}^{V}}^{\sigma(\hat{s}, \cdot)}\left(\hat{y}, I_{V}\right)\right) \\
& \leqq \omega_{K}(|\hat{t}-\hat{s}|) .
\end{aligned}
$$

This implies

$$
\left(\gamma+\gamma^{\prime}\right) / T^{2} \leqq C(1+K)\left|\Lambda_{\chi_{l}^{V} \chi_{r}^{V}}^{\sigma(\hat{t}, \cdot)}\left(\hat{x}, I_{U}\right)-\Lambda_{\chi_{l}^{V} \chi_{r}^{V}}^{\sigma(\hat{s}, \cdot)}\left(\hat{y}, I_{V}\right)\right|+\omega_{K}(|\hat{t}-\hat{s}|)
$$

by (FL). By Theorem 8(iii), we know $I_{U}=I_{V}+\hat{x}-\hat{y}$. Sending $\varepsilon$ to zero, we observe that $\hat{x}-\hat{y} \rightarrow 0$ by Proposition 5 (ii). By (S), we know that $\sigma_{x}(s, \cdot)$ is uniformly bounded. We now invoke continuity results (Theorems 2 and 3 (ii)) to get

$$
\begin{aligned}
& \Lambda_{\chi_{l}^{V} \chi_{r}^{V}}^{\sigma(\hat{t}, \cdot)}\left(\hat{x}, I_{U}\right) \rightarrow \Lambda_{\chi_{l}^{V} \chi_{r}^{V}}^{\sigma(\bar{t}, \cdot)}(\bar{x}, I), \\
& \Lambda_{\chi_{l}^{V} \chi_{r}^{V}}^{\sigma(\hat{s}, \cdot)}\left(\hat{y}, I_{V}\right) \rightarrow \Lambda_{\chi_{l}^{V} \chi_{r}^{V}}^{\sigma(\bar{s}, \cdot)}(\bar{x}, I)
\end{aligned}
$$

as $\varepsilon \rightarrow 0$, where $\bar{x}(=\bar{y}), \bar{t}, \bar{s}$ is a subsequent limit of $\hat{x}, \hat{y}, \hat{t}, \hat{s}$ as $\varepsilon \rightarrow 0$ and $I$ is a subsequent limit of $I_{U}$ which is the same as the limit of $I_{V}$. Note that $U$ and $V$ depend $\varepsilon$, so do $I_{U}$ and $I_{V}$. However, the convergence is uniform with respect to the interval and $\sigma$, so we are able to obtain (4.8). Applying Theorem 2 and Theorem 3(ii) again to (4.8), we let $\delta \rightarrow 0$ and observe that the right hand sides of (4.8) converge to the same value. We now send $\varepsilon \rightarrow 0$ and then $\delta \rightarrow 0$ in (4.7) to get $\left(\gamma+\gamma^{\prime}\right) / T^{2} \leqq 0$, which is a contradiction.

Case II is rather standard [11,16,17]. The assumptions (FL) and (S) are useful in this step. Case III is essentially the same as Case I (or even easier) if one admits the zero curvature lemma (Lemma 2).

\subsection{Periodic Version}

As noted in [17] a similar argument yields the comparison principle under spatially periodic boundary conditions. In fact, the argument is even simpler because there is no lateral boundary of $Q=(0, T) \times \mathbf{T}, \mathbf{T}=\mathbf{R} / \omega \mathbf{Z}, \omega>0$. For the reader's convenience, we state the comparison principle for the periodic boundary condition. 
Theorem 9 (Comparison). Let us assume that the conditions $(W),(S),(F 1),(F 2)$, $(F L)$ and $(F T)$ hold and in addition set $P$ is finite. Let $u$ and $v$ be respectively sub- and supersolutions of (3.1) in $Q=(0, T) \times \mathbf{T}, \mathbf{T}=\mathbf{R} / \omega \mathbf{Z}$ with period $\omega$. If $u^{*} \leqq v_{*}$ at $t=0$, then $u^{*} \leqq v_{*}$ in $Q$.

Remark 3. As usual, Theorems 7 and 9 can be extended to the case when $F=$ $F(u, t, p, X)$ depends explicitly also on $u$, provided that $u \mapsto F(u, t, p, X)+$ $k u=: \tilde{F}$ is nondecreasing for some $k \geqq 0$ and $\tilde{F}$ is continuous as a function of $(u, t, p, X)$. Of course, assumptions (FL) and (FT) should be uniform for all $u$ with $|u| \leqq K$ for a given $K$. If $k=0$, the proof is the same except for the trivial modification to the way of comparing (4.5) and (4.6). If $k>0$, we have to introduce a new variable $\tilde{u}=u \exp (-k t)$ and reduce the problem to the case $k=0$. Note that, differently from the standard case [16], when singularity set $P$ is empty, our singular set (jump discontinuity) for $\tilde{u}_{x}$ depends on time, which apparently yields an extra difficulty. However, we are able to circumvent this difficulty by using old variables to calculate $\Lambda$ and the slope, while using new variables $\tilde{u}$ and $\tilde{v}$ to find a maximizer of $\Phi$.

\section{Examples of Solutions}

In $[27,29,30]$ we constructed variational solutions to

$$
\beta V-\kappa_{\gamma}=\sigma,
$$

while increasing generality of the setting, where $\beta=M^{-1}$ is the kinetic coefficient. We considered graphs, possibly satisfying an additional boundary condition, and simple closed Lipschitz curves, which we called bent rectangles. We will show that the variational solutions to (5.1) for the evolution of graphs are viscosity solutions in the sense of the present paper. For the sake of illustration the theory, we will not consider the general case of [27] but only simple ones presented in [29]. To be precise, we are going to deal here with a simplification of the case studied in [29], where we investigated graphs of functions defined over a finite interval $J$. We considered solutions having exactly three facets and two of them touched the boundary at the right angle. Here, we study a graph over $\mathbf{R}$, with some restrictions on the data.

We expect that the results of the present paper may be applied to closed curves, but we will not elaborate upon this.

The advantage of studying graphs in the parametric approach is that the set of parameters is independent of time. Thus, the main difficulty is interpreting (5.1) in a local coordinate system. We present the setting after [29].

We specify the surface energy density (or anisotropy function) by the formula

$$
\gamma\left(p_{1}, p_{2}\right)=\left|p_{1}\right| \gamma_{\Lambda}+\left|p_{2}\right| \gamma_{T}, \gamma_{\Lambda}, \gamma_{T}>0
$$

we assume a simplifying form of the kinetic coefficient $\beta=1 / M$

$$
\beta\left(n_{1}, n_{2}\right)=\frac{1}{\max \left(\left|n_{1}\right|,\left|n_{2}\right|\right)},
$$

for $n_{1}^{2}+n_{2}^{2}=1$. Subsequently, $\beta$ is extended by 1 -homogeneity to $\mathbf{R}^{2}$. 


\subsection{Graphs over $\mathbf{R}$}

We consider the evolution of graph $\Gamma(t)=\left\{(x, y) \in \mathbf{R}^{2}: y=d(t, x)\right\}$, where $d(t, \cdot): \mathbf{R} \rightarrow \mathbf{R}_{+}$. For the sake of simplicity we assume that function $d(t, \cdot)$ is admissible (in $x$ ) for all $t \geqq 0$. We shall say that function $d$ is admissible provided that:

(a) $d$ is Lipschitz continuous;

(b) $d$ is even;

(c) it is bounded;

(d) $\left(\lambda_{0},+\infty\right) \ni x \mapsto d(x)$ is strictly increasing, for a positive $\lambda_{0}$;

(e) $\left\{d_{x}=0\right\}=\left(-\lambda_{0}, \lambda_{0}\right)$.

The last condition means that we consider a simple yet nontrivial case when $d$ has exactly one faceted region. We stress, however, that facet $\left(-l_{0}, l_{0}\right)$ may be strictly included in $\left(-\lambda_{0}, \lambda_{0}\right)$. This results from solving the minimization problem with constraints, see Fig. 3 below.

We have to explain the definition of $\kappa_{\gamma}$. Formally,

$$
\kappa_{\gamma}=-\operatorname{div}_{S}\left(\nabla_{\zeta} \gamma(\mathbf{n})\right)
$$

where $\mathbf{n}$ is the outer normal to $\Gamma$ and for $\gamma$, given by (5.3), we have,

$$
\nabla \gamma\left(p_{1}, p_{2}\right)=\left(\gamma_{\Lambda} \operatorname{sgn}\left(p_{1}\right), \gamma_{T} \operatorname{sgn}\left(p_{2}\right)\right) .
$$

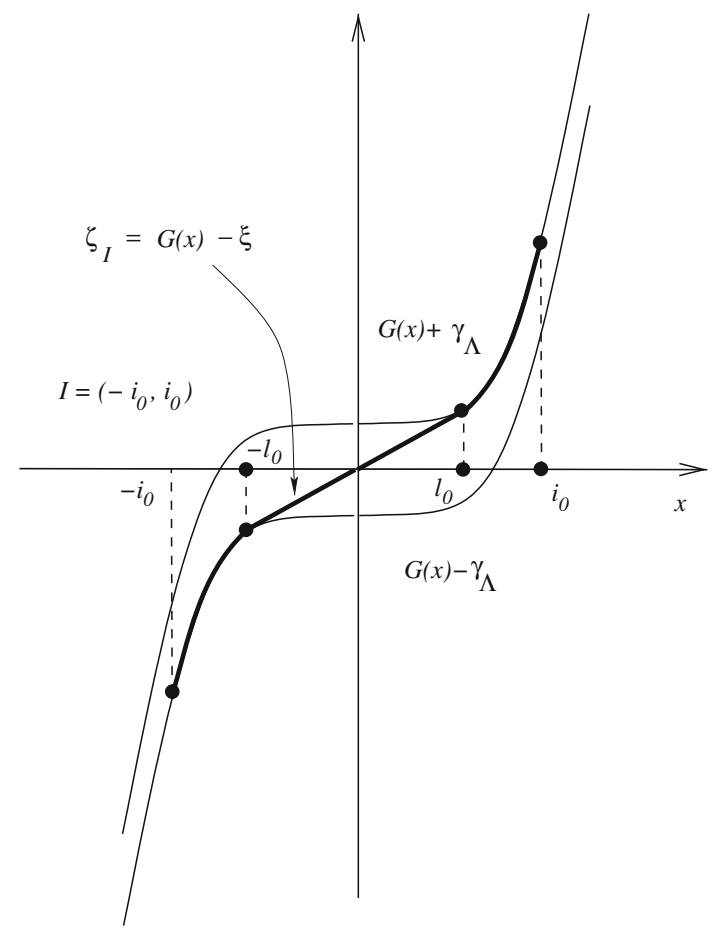

Fig. 3. Graph of $G-\xi$ restricted to $I \supset\left(-l_{0}, l_{0}\right)$ 
In the present case $\mathbf{n}=\left(-d_{x}, 1\right) / \sqrt{1+d_{x}^{2}}$. Thus, we immediately obtain

$$
\frac{\beta(\mathbf{n}) d_{t}}{\sqrt{1+d_{x}^{2}}}=\sigma+\gamma_{\Lambda} \frac{\partial}{\partial x}\left(\frac{\mathrm{d}}{\mathrm{d} p_{1}}\left|d_{x}\right|\right) .
$$

This is exactly equation (1.1) with $W\left(p_{1}\right)=\gamma_{\Lambda}\left|p_{1}\right|$ and $a\left(p_{1}\right)=\max \left\{\left|p_{1}\right|, 1\right\}$, hence our theory applies.

In [29] we interpreted (5.1) differently. Namely, we replaced gradient $\nabla_{\zeta} \gamma$, which is defined only almost everywhere by the subdifferential, $\partial_{\zeta} \gamma$, which is well defined for all $p \in \mathbf{R}^{2}$, because $\gamma$ is convex. However, we had to consider sections $\xi$ of the subdifferential, that is $\xi(x) \in \partial_{\zeta} \gamma(\mathbf{n}(x))$. That is here, where we change notation as compared with the Introduction and Section 2. In the Introduction our present $\xi$ was denoted by $\eta$. On the other hand, writing $\xi(x) \in \partial_{\zeta} \gamma(\mathbf{n}(x))$ is consistent with the papers that are the source of our examples.

As a result, we end up with

$$
\frac{\beta(\mathbf{n}) d_{t}}{\sqrt{1+d_{x}^{2}}}=\sigma-\tau \cdot \frac{\partial \xi}{\partial \tau},
$$

where $\tau$ is a unit tangent, (see [29, eq. (2.3)]).

In order to select $\xi$ we introduce a functional

$$
\mathcal{E}(\xi)=\frac{1}{2} \int_{\Gamma(t)}\left|\sigma-\operatorname{div}_{S} \xi\right|^{2} \mathrm{~d} \mathcal{H}^{1}
$$

defined over $\mathcal{D}$,

$$
\mathcal{D}=\left\{\xi \in L^{\infty}(\Gamma): \xi(x) \in \partial \gamma(\mathbf{n}(x)), \operatorname{div}_{S} \xi \in L^{2}(\Gamma)\right\} .
$$

The graph of $\Gamma(t)$ has the infinite one-dimensional Hausdorff measure. But condition $\operatorname{div}_{S} \xi \in L^{2}(\Gamma)$ does not introduce additional unexpected restrictions, because outside of the facets we have $\xi=\nabla \gamma(\mathbf{n})$, where $\mathbf{n} \neq \mathbf{n}_{\Lambda}, \mathbf{n}_{R}$ and $\mathbf{n}_{\Lambda}=(1,0)$, $\mathbf{n}_{R}=(0,1)$.

We call a couple $(\Gamma, \xi)$ a variational solution to (5.1), provided that $\Gamma$ is the graph of an admissible function $d$, as described above, and at each time instant $t$, the vector field $\xi(t, \cdot): \Gamma \rightarrow \mathbf{R}^{2}$ is a minimizer of $\mathcal{E}$, that is

$$
\mathcal{E}(\xi)=\min \{\mathcal{E}(\zeta): \zeta \in \mathcal{D}\}
$$

We can show that under natural conditions on $\sigma$, equation (5.1) takes a form which is suitable for the analysis.

We notice that if $\xi$ is a solution to (5.6), then the boundary of the coincidence set $\pm l_{0}$ need not coincide with boundary of the flat region $\pm \lambda_{0}$, postulated by the definition of the admissible function, thus $l_{0} \leqq \lambda_{0}$. For the sake of notational simplicity, we shall write

$$
R_{0}:=\left.d\right|_{\left(-l_{0}, l_{0}\right)} .
$$

Once we settle the notation, we establish the following fact. 
Proposition 8. We assume that $\sigma, \sigma_{x}, \in C\left(\mathbf{R}_{+} \times \mathbf{R}\right)$ and $\sigma$ satisfies the following conditions:

$$
\sigma(t,-x)=\sigma(t, x), \quad x \frac{\partial \sigma}{\partial x}(t, x)>0, \text { for } \quad x \neq 0 .
$$

Let us suppose that $(\Gamma, \xi)$ is a variational solution to (5.1), where $\Gamma=\Gamma(d)$ is the graph of $d$, such that at each time instant $t \geqq 0 d(t, \cdot)$ has exactly one faceted region, $\left(-l_{0}, l_{0}\right)$. Furthermore, for all $t \geqq 0$ function $d(t, \cdot)$ is piecewise $C^{1}$. Then,

(a) We have the following formula for $\xi_{1}$ for each time $t \geqq 0$

$\xi_{1}(t, x)= \begin{cases}x\left(f_{0}^{x} \sigma(t, s) \mathrm{d} s-f_{0}^{l_{0}} \sigma(t, s) \mathrm{d} s\right)-\frac{x}{l_{0}} \gamma\left(\mathbf{n}_{\Lambda}\right) & \text { for } \quad x \in\left[0, l_{0}\right) \\ -\gamma\left(\mathbf{n}_{\Lambda}\right) & \text { for } \quad x \in\left[l_{0}, \infty\right)\end{cases}$

where we write $f_{A} f \mathrm{~d} \mu=\frac{1}{\mu(A)} \int_{A} f \mathrm{~d} \mu$. In addition, $\dot{R}_{0}>0$.

(b) Equation (5.1) (and hence (5.5)) takes the following form,

$$
\begin{aligned}
& \dot{R}_{0}=f_{0}^{l_{0}} \sigma(t, s) \mathrm{d} s+\frac{\gamma\left(\mathbf{n}_{\Lambda}\right)}{l_{0}} \text { on }\left(-l_{0}, l_{0}\right) ; \\
& d_{t}=\sigma \quad \text { on }\left[l_{0}, \infty\right) .
\end{aligned}
$$

Remark 4. The above result is based upon [29, Proposition 2.5], [30, Proposition 3.2] derived for graphs over $[-L, L]$ having three facets, two of them touching the boundary of $[-L, L]$. In the absence of the additional facets the argument gets simpler than in [29] and [30] and it is omitted.

Let us warn the reader that we use the notion 'faceted region' in the sense defined in the present paper. In [29] and [30] its meaning is different.

It turns out that $l_{0}(\cdot)$ is a genuine free boundary. We obviously need information about its behavior. Without it, the above system is not closed.

Let us suppose that $t \geqq 0$. The necessary and sufficient condition for continuity of the function given below

$$
\chi_{\left[0, l_{0}(t)\right]} R_{0}(t)+\chi_{\left(l_{0}(t), \infty\right)} d(t, x),
$$

is the following matching condition

$$
R_{0}(t)=d\left(t, l_{0}\right) .
$$

In addition, since we have a faceted region, the coincidence set of the obstacle problem (5.6) may not be empty. By definition, $\pm l_{0}$ forms its boundary, that is, $l_{0} \leqq \lambda_{0}$, then at such a point

$$
\frac{\partial \xi}{\partial x}\left(l_{0}\right)=0 .
$$

We shall say that $(\Gamma, \xi)$ satisfies the tangency condition at $l_{0}$.

However, if $d_{x}^{+}\left(l_{0}(t), t\right)>0$, then we just have a boundary condition at this point and (5.11) does not hold.

We have the following two existence results. 
Theorem 10. Let us assume (5.2) and consider system (5.9), augmented with initial condition $\left(\Gamma_{0}, \xi_{0}\right)$, where

$$
\Gamma_{0}=\left\{(x, y) \in \mathbf{R}^{2}: x \in \mathbf{R}, y=d_{0}(x)\right\},
$$

$d_{0}$ is an admissible function, satisfying $\left|d_{0, x}(x)\right|<1$ for all $x \in \mathbf{R}$. In particular, real, positive numbers $l_{00} R_{00}=\left.d\right|_{\left(-l_{00}, l_{00}\right)}$ are given. We assume that $\sigma$ satisfies (5.7). Moreover, we impose the following conditions:

(a) $d_{0} \in C^{1}\left(\mathbf{R} \backslash\left(-l_{00}, l_{00}\right)\right)$ and for all $x \in \mathbf{R} \backslash\left(-l_{00}, l_{00}\right)$ the derivative $d_{0, x}$ is different from zero;

(b) there is exactly one faceted region of $d_{0}$, where $\Gamma(0)=\Gamma\left(d_{0}\right)$, namely it is $\left(-l_{00}, l_{00}\right)$

(c) the matching condition (5.10) holds at $t=0$, that is $R_{00}=d_{0}\left(l_{00}\right)$;

(d) the tangency condition (5.11) is satisfied at $t=0$, that is

$$
\sigma\left(0, l_{00}\right)=f_{0}^{l_{00}} \sigma(0, s) \mathrm{d} s+\frac{\gamma\left(\mathbf{n}_{\Lambda}\right)}{l_{00}},
$$

(e)

$$
\Sigma_{0}=f_{0}^{l_{00}} \sigma_{t}(0, y) \mathrm{d} y-\sigma_{t}\left(0, l_{00}\right)<0 .
$$

Then,

(i) There exists a unique local in time solution to (5.9), $R_{0}$ and $d(t, \cdot) \in$ $C^{1}\left(\left(-\infty,-l_{0}\right] \cup\left[l_{0}, \infty\right)\right)$ and $d(t, \cdot)$ is strictly increasing and its derivative $d_{x}(t, x)$ never vanishes for $x \in \mathbf{R} \backslash\left(-l_{0}(t), l_{0}(t)\right)$;

(ii) The matching (5.10) and tangency (5.11) conditions hold for all times $t>0$, that is if we extend $d(t, \cdot)$ to $\mathbf{R}$ by

$$
\bar{d}(t, x)=\left\{\begin{array}{lll}
d(t, x) & \text { if } & |x| \in\left[l_{0}, \infty\right) \\
R_{0}(t) & \text { if } & |x| \in\left[0, l_{0}\right),
\end{array}\right.
$$

then $\bar{d}(t, \cdot)$ is Lipschitz continuous on $\mathbf{R}$ (subsequently we drop the bar over the extension).

(iii) If $\xi_{1}(t, x)$ is given by formula (5.8) for $x>0$ and we set $\xi_{1}(t, x)=-\xi_{1}(t,-x)$ for $x<0$, then $(\Gamma(d(t, \cdot)), \xi(t, \cdot))_{t \in[0, T)}$ is a variational solution to (5.1), provided that $\xi(t, \cdot)=\left(\xi_{1}(t, \cdot), \gamma\left(\mathbf{n}_{R}\right)\right)$.

Remark 5. Let us stress again that $l_{00}$ is defined as the boundary of the coincidence set

$$
\left\{x:\left|\xi_{1}(x)\right|=\gamma_{\Lambda}\right\},
$$

where $\xi$ is a solution to variational problem (5.6). We note that in general

$$
\left[-l_{0}, l_{0}\right] \subset\left\{x: d_{x}(t, x)=0\right\}
$$

and the inclusion may be strict. 
Theorem 11. Let us suppose that all the assumptions of Theorem 10 hold, except (d), that is tangency condition (5.11) and the inequality sign in (e) are reversed, that is we have

$$
\Sigma_{0}>0
$$

Instead of (5.11) the following inequality is satisfied

$$
\sigma\left(0, l_{00}\right)-f_{0}^{l_{00}} \sigma(0, s) \mathrm{d} s+\frac{\gamma\left(\mathbf{n}_{\Lambda}\right)}{l_{00}}<0 .
$$

Moreover, we assume that $d_{0} \in C^{1,1}\left(\left[l_{00}, \infty\right)\right)$, the right derivative $d_{x}^{+}\left(0, l_{00}\right)$ is positive and $\sigma \in C^{1,1}$. Then, there is a unique local in time solution to (5.9), such that at no time $t>0$ tangency condition (5.11) holds. Subsequently, if $\xi(t, \cdot)$ is defined, as in Theorem 10, (iii), then $(\Gamma(d(t, \cdot), \xi(t, \cdot))$ is a variational solution to (5.1).

Remark 6. We note that $l_{0}$ is a genuine free boundary; its behavior is determined by $\sigma$. For instance if $\sigma$ is independent of time and $\sigma=\sigma(x)$, then $l_{0}(t)=l_{00}$. The type of behavior of the interfacial curve is determined by $\Sigma_{0}$, this quantity is defined by [30, eq. (3.14)] and the properties of $l_{0}$ are presented in [30, Proposition 3.4].

These two Theorems are based upon [29, Theorem 2.10] and the analysis of [30, Section 3.1]. The present statements are easier than the original ones in [29, Theorem 2.10] and in [30, Section 3.1], because we deal with a single facet for a graph of an admissible function, but the main difference is that here we have an unbounded domain. For the sake of completeness, we offer a sketch of the proof in the Appendix.

\subsection{Variational Solutions are Viscosity Solutions and They are Unique}

Here, we shall see that our variational solution over $\mathbf{R}$ can be regarded as the viscosity solutions. Hence, they will be unique. The comparison principle has been shown for equations on a bounded domain, but our sub- and supersolutions are fully determined for large values of $|x|$, thus a comparison principle for bounded $|x|$ is sufficient. We will explain it in Corollary 2 following Theorem 12.

Theorem 12. Under the conditions specified above, the variational solutions, constructed in Theorem 10 and in Theorem 11, are viscosity solutions in the sense of the present paper, as long as $\left|d_{x}\right| \leqq 1$.

Proof. Of course equation (5.4), augmented with the initial condition, may be written as

$$
\left\{\begin{array}{l}
d_{t}-a\left(d_{x}\right) \Lambda_{W}^{\sigma}(d)=0 \\
d(x, 0)=d_{0}(x)
\end{array}\right.
$$

where $\Lambda_{W}^{\sigma}(d)=\frac{\mathrm{d}}{\mathrm{d} x} \zeta_{\chi_{l} \chi_{r}}$ is given by (2.5) and the signs of $\chi_{r}, \chi_{l}$ depend upon the point we are considering. We will show that if $(\Gamma(d), \xi)$ is a variational solution, 
then $\Lambda_{W}^{\sigma}(d)=\sigma-\frac{\partial \xi}{\partial x}$, where $\xi$ is given by (5.8) in Proposition 8. The interval $\left(-l_{0}, l_{0}\right)$ is the inverse image of a faceted region of $\Gamma$ in the language of $[29,30]$, it is the faceted region in the present paper sense. If $I$ is any interval containing $\left(-l_{0}, l_{0}\right)$, then $\bar{\xi}=\left.\xi\right|_{I}$ is a solution to the minimization problem,

$$
\min \left\{\mathcal{E}_{I}(\zeta): \zeta \in \mathcal{D}_{I}\right\}
$$

We write, $\Gamma_{I}(t)=\{(x, y) \in \Gamma(t): x \in I\}$ and

$$
\begin{aligned}
\mathcal{E}_{I}(\zeta) & =\frac{1}{2} \int_{\Gamma_{I}(t)}\left|\sigma-\operatorname{div}_{S} \zeta\right|^{2} d \mathcal{H}^{1} \\
\mathcal{D}_{I} & =\left\{\zeta \in L^{\infty}\left(\Gamma_{I}\right): \zeta(x) \in \partial \gamma(\mathbf{n}(x)), \operatorname{div}_{S} \zeta \in L^{2}\left(\Gamma_{I}\right), \zeta=\left.\xi\right|_{\partial I}\right\} .
\end{aligned}
$$

Indeed, if there existed $\zeta_{I}$, a solution to (5.12), such that $\mathcal{E}_{I}\left(\zeta_{I}\right)<\mathcal{E}_{I}\left(\xi_{I}\right)$, then this indicates that $\xi$ is not a solution to (5.6), which is not possible. Thus, if $\left(-l_{0}, l_{0}\right) \subset I$, then $\pm l_{0}$ form the boundary of the coincidence set, where the solution $\xi$ to (5.6) attains $-\gamma_{\Lambda}$, that is on the coincidence set $\zeta_{I}(x)=G(x)+\gamma_{\Lambda}$. Here, $G(x)$ denotes $\int_{0}^{x} \sigma(s) \mathrm{d} s$. The situation is illustrated in Fig. 3 above.

We have to justify the possibility of taking the boundary conditions in the definition of $\mathcal{D}_{I}$. We know that $\xi$ is a solution to the obstacle problem (5.6) and $\left(-\infty,-l_{0}\right) \cup\left(l_{0}, \infty\right)$ is the coincidence set. Using the argument of the proof of [29, Proposition 2.5], [30, Proposition 3.2], one can show that $\left.\xi\right|_{\left(-\infty,-l_{0}\right]}=\gamma_{\Lambda}$ and $\left.\xi\right|_{\left[l_{0}, \infty\right)}=-\gamma_{\Lambda}$. Thus, $\xi$ restricted to each connected component of the $I \backslash\left[-l_{0}, l_{0}\right]$ is constant.

Let us now calculate $\Lambda_{W}^{\sigma}$. For points of the coincidence set, it is clear that $\Lambda_{W}^{\sigma}=\sigma$, as desired. Let us consider interval $\left[-l_{0}, l_{0}\right]$. By the definition, see (2.5), $\Lambda_{W}^{\sigma}=\frac{\mathrm{d}}{\mathrm{d} x} \zeta_{\chi_{l} \chi_{r}, I}$, where $\zeta_{\chi_{l} \chi_{r}, I}$ is a solution to the following obstacle problem,

$$
\min \left\{J_{\chi_{l} \chi_{r}}^{Z}(\omega, I): \omega \in K_{\chi_{l} \chi_{r}}^{Z}\right\}
$$

where $Z(x)=\int_{0}^{x} \sigma(t, s) \mathrm{d} s$ and for $\left[-l_{0}, l_{0}\right]$, we have $\chi_{l}=+1=\chi_{r}$,

$$
\begin{aligned}
K_{++}^{Z}= & \left\{\omega \in H^{1}\left(-l_{0}, l_{0}\right): Z(x)-\gamma_{\Lambda} \leqq \omega(x) \leqq Z(x)+\gamma_{\Lambda}, \omega\left( \pm l_{0}\right)=Z\left( \pm l_{0}\right)\right. \\
& \left. \pm \gamma_{\Lambda}\right\} .
\end{aligned}
$$

Since the boundary conditions in $K_{++}^{Z}$ are that of $\mathcal{D}_{\left[-l_{0}, l_{0}\right]}$, we immediately conclude, by previous considerations, that $\zeta$ defined by $Z-\xi$ is the solution to (5.13). Hence, $\Lambda_{W}^{\sigma}=\sigma-\frac{\partial \xi}{\partial x}$.

After these preparations, we may check that a variational solution is a viscosity solution. First, we shall see that $d$ is a supersolution. For this purpose, we take a test function $\varphi \in A_{P}(Q)$, such that $d-\varphi$ attains a minimum at $\left(x_{0}, t_{0}\right)$, where $t_{0} \in(0, T)$. We have to show that

$$
\varphi_{t}-\Lambda_{W}^{\sigma} \geqq 0 .
$$

Inequality (5.14) (and (5.16) below) is to be checked at each point. We have to consider two cases for the interfacial curves: (a) the free boundary $l_{0}$ is a tangency 
curve; (b) the free boundary $l_{0}$ is a matching curve and the tangency condition is violated.

In addition, in the course of proving (5.14), we will consider three cases separately:

(i) $\left|x_{0}\right|>l_{0}\left(t_{0}\right)$, (ii) $\left|x_{0}\right| \in\left[0, l_{0}\left(t_{0}\right)\right)$, (iii) $\left|x_{0}\right|=l_{0}\left(t_{0}\right)$.

We begin with (i). Since we assumed that $d_{0} \in C^{1}$, we know (see Theorem 10 or Theorem 11) that at $\left(x_{0}, t_{0}\right)$ function $d$ is differentiable. Hence, for $\varphi(x, t)=$ $f(x)+g(t)$ with $d-\varphi \geqq 0$ in a neighborhood of $\left(x_{0}, t_{0}\right)$, we have

$$
d_{x}\left(x_{0}, t_{0}\right)=f^{\prime}\left(x_{0}\right), \quad d_{t}\left(x_{0}, t_{0}\right)=g^{\prime}\left(t_{0}\right) .
$$

Due to Definition 2, we have $\Lambda_{W}^{\sigma}(\varphi)=\sigma=\Lambda_{W}^{\sigma}(d)$. As a result,

$$
0=d_{t}-\sigma=g^{\prime}-\Lambda_{W}^{\sigma}(\varphi)=\varphi_{t}-\Lambda_{W}^{\sigma}(\varphi),
$$

as desired.

Now, we look at (ii). The argument depends on the type of the interfacial curve $l_{0}$. Let us first assume that $l_{0}$ is a tangency curve.

In the considered case, $d$ is also differentiable at $\left(x_{0}, t_{0}\right)$. If $\varphi$ is a test function, such that $d-\varphi$ attains its minimum at $\left(x_{0}, t_{0}\right)$, then

$$
d_{x}\left(x_{0}, t_{0}\right)=0=f^{\prime}\left(x_{0}\right), \quad d_{t}\left(x_{0}, t_{0}\right)=g^{\prime}\left(t_{0}\right) .
$$

Since $f \in C_{P}^{2}(\Omega)$, we immediately see that $I=R\left(f, x_{0}\right)$, the faceted region of $\varphi$ at $\left(x_{0}, t_{0}\right)$, must contain $\left[-l_{0}, l_{0}\right]$. Let us suppose that $\xi_{I}$ is the solution to

$$
\min \left\{\mathcal{E}_{I}(\omega): \omega \in \mathcal{D}_{I}\right\}
$$

By the geometric interpretation of the obstacle problem (5.6), [29, Proposition 2.3], the coincidence set is $I \backslash\left(-l_{0}, l_{0}\right)$. This is the place where we use the fact that the tangency condition holds at $x_{0}$.

As a result of the above observation, we have $\Lambda_{W}^{\sigma}(d)=\Lambda_{W}^{\sigma}(\varphi)$. Moreover,

$$
\begin{aligned}
\Lambda_{W}^{\sigma}(d) & =\sigma-\frac{\partial \xi}{\partial x} \\
& =f_{0}^{l_{0}} \sigma(t, s) \mathrm{d} s+\frac{\gamma\left(\mathbf{n}_{\Lambda}\right)}{l_{0}} .
\end{aligned}
$$

Thus, by (5.9)

$$
0=\dot{R}_{0}-f_{0}^{l_{0}\left(t_{0}\right)} \sigma(s) \mathrm{d} s-\frac{\gamma\left(\mathbf{n}_{\Lambda}\right)}{l_{0}\left(t_{0}\right)}=d_{t}-\Lambda_{W}^{\sigma}(d)=\varphi_{t}-\Lambda_{W}^{\sigma}(\varphi),
$$

as desired.

Let us note that this argument works well for $\left(x_{0}, t_{0}\right)=\left(l_{0}\left(t_{0}\right), t_{0}\right)$ if the tangency condition holds, so (iii) holds in this case.

We continue our analysis of case (ii). We have to consider the situation when $l_{0}$ is a matching curve. We will have to compare $\Lambda_{W}^{\sigma}(d)$ and $\Lambda_{W}^{\sigma}(\varphi)$. One way is to invoke Theorem 4 , but we think it is instructive to check it directly. 
Let us suppose that $I=[-a, b]$ is the faceted region of $\varphi$ containing $\left(x_{0}, t_{0}\right)$. We consider the minimization problem (5.13) defining $\zeta_{I}$ on that interval. Without the loss of generality, we may restrict our attention to a subinterval $\left[\mu_{0}, \mu_{1}\right] \subset[-a, b]$, such that $\frac{\mathrm{d} \zeta_{I}}{\mathrm{~d} x}$ is constant on $\left[\mu_{0}, \mu_{1}\right]$. Let us first consider that situation when $\mu_{0}=-\mu_{1}$. We have to compare velocities $\frac{\mathrm{d} \zeta_{I}}{\mathrm{~d} x}$ and $\frac{\mathrm{d} \xi}{\mathrm{d} x}$ on $\left[-l_{0}, l_{0}\right]$. Since the tangency condition is violated at $l_{0}$, then there is a possibility of bigger faceted regions containing $\left[-l_{0}, l_{0}\right]$. Moreover, $\frac{\mathrm{d} \zeta_{I}}{\mathrm{~d} x}$ is a slope of a line connecting 0 and $Z\left(\mu_{1}\right)+\gamma_{\Lambda}$, while $\frac{\mathrm{d} \xi}{\mathrm{d} x}$ is a slope of a line connecting 0 and $Z\left(l_{0}\right)+\gamma_{\Lambda}$. Since $Z$ is strictly increasing, we deduce that $\frac{\mathrm{d} \zeta_{I}}{\mathrm{~d} x}<\frac{\mathrm{d} \xi}{\mathrm{d} x}$. The same observation applies when we want to compare slopes of minimizers to $(5.13)$ on $[-a, b]$ and $\left[-\mu_{1}, \mu_{1}\right]$ and $a=\mu_{1}$ or $b=\mu_{1}$ but $[-a, b] \supset\left[-\mu_{1}, \mu_{1}\right]$. Thus, we have

$$
\begin{aligned}
\varphi_{t}-\Lambda_{W}^{\sigma}(\varphi) & \geqq d_{t}-\Lambda_{W}^{\sigma}(d) \\
& =\dot{R}_{0}-f_{0}^{l_{0}} \sigma(t, s) \mathrm{d} s-\frac{\gamma\left(\mathbf{n}_{\Lambda}\right)}{l_{0}} \\
& =0 .
\end{aligned}
$$

(iii) In order to complete the discussion of the facet, we have to consider the case when at the interfacial point the tangency condition is violated. Let us suppose that this happens at $x_{0}=l_{0}$ (the case $x_{0}=-l_{0}$ is analogous). At this point $d\left(t_{0}, x_{0}\right)$ need not be differentiable with respect to $x$. Hence, if $\varphi$ is a test function such that $d-\varphi$ attains its minimum, then $d_{x}^{-}\left(l_{0}\left(t_{0}\right), t_{0}\right)=0$ and $d_{x}^{+}\left(l_{0}\left(t_{0}\right), t_{0}\right) \geqq 0$.

The point $\left(l_{0}\left(t_{0}\right), t_{0}\right)$ belongs to the faceted region of $d$, hence it belongs to the faceted region of the test function $\varphi$. As a result, the above consideration on $\Lambda_{W}^{\sigma}(\varphi)$ is valid. Hence, the series of inequalities (5.15) is valid too.

We also have to check that $d$ is a subsolution. For this purpose we take a test function $\varphi \in A_{P}(Q)$, such that

$$
\max (d-\varphi)=d\left(t_{0}, x_{0}\right)-\varphi\left(t_{0}, x_{0}\right) .
$$

We shall show that

$$
\varphi_{t}-\Lambda_{W}^{\sigma} \leqq 0
$$

We consider the same three cases. They are handled in an analogous way, we exploit the fact that $d(t, \cdot)$ is a $C^{1}$ function on $\left(-l_{0}, l_{0}\right)$ and on $\mathbf{R} \backslash\left[-l_{0}, l_{0}\right]$.

The case (i) is handled as before, because of differentiability of $d$ and $\varphi$ at $\left(x_{0}, t_{0}\right)$.

(ii) If $\left|x_{0}\right|<l_{0}(t)$, then the faceted region of $\varphi$ is contained in $\left[-l_{0}\left(t_{0}\right), l_{0}\left(t_{0}\right)\right]$. By the previous analysis, we conclude that $\Lambda_{W}^{\sigma}(\varphi) \geqq \Lambda_{W}^{\sigma}(d)$. Hence,

$$
\varphi_{t}-\Lambda_{W}^{\sigma}(\varphi) \leqq d_{t}-\Lambda_{W}^{\sigma}(d)=0 .
$$

Case (iii) is handled in a completely analogous way, as before. We omit the details.

Corollary 2. Let us suppose that the assumptions of Theorem 12 hold. The variational solutions constructed in Theorems 10 and 11 are unique, as long as $\left|d_{x}\right| \leqq 1$ and the initial condition $d_{0}$ is strictly increasing on $\left[l_{00}, \infty\right)$. 
Proof. Let us suppose that $\left(\Gamma\left(d^{i}\right), \xi^{i}\right)$ are two variational solutions, with initial data $\Gamma\left(d_{0}\right)$, where $d_{0}$ is admissible. We notice that it is sufficient to show that $d^{1}=d^{2}$.

Let us set $A=\max _{t \in[0, T)} l_{0}(t)+1$. Due to (5.7), by formula (6.2), we conclude that $d_{x}^{i}(t, x) \neq 0$ for all $(t, x) \in(0, T) \times(A, \infty)$. Since we solve an ODE for $|x|>A$, by the inspection of equation (6.1) we immediately see that if $v:=d^{1}$ is a supersolution and $u:=d^{2}$ is a subsolution to (6.1), then $v \geqq u$. Subsequently, by interchanging the roles of $d^{1}$ and $d^{2}$, we conclude that $d^{1}=d^{2}$ for $(t, x) \in$ $[0, T) \times \mathbf{R} \backslash(-A, A)$. As a result, we can see that an application of the Comparison Principle on $(-A, A)$ yields that $d^{1}=d^{2}$ for all $(t, x) \in[0, T) \times \mathbf{R}$.

Acknowledgments All authors thank the referee for his/her insightful comments which helped to improve the readability of this paper. The second author was in a part supported by the Japan Society for the Promotion of Science (JSPS) through the grants for scientific research Kiban (S) 21224001, Kiban (A) 23244015 and Houga 25610025. The third author was in part supported by the Polish Ministry of Science grant NN 201 268935. During the preparation of this manuscript PR was enjoying the hospitality of Hokkaido University, which is gratefully acknowledged.

Open Access This article is distributed under the terms of the Creative Commons Attribution Noncommercial License which permits any noncommercial use, distribution, and reproduction in any medium, provided the original author(s) and source are credited.

\section{Appendix}

Here we give a sketch of proof of Theorems 10 and 11 by pointing to the main differences with [29, Theorem 2.10] and [30, Section 3.1].

In [29], we considered equation (5.5) on a bounded interval $J$. The initial condition, hence the solution, had three facets, two of them touching the endpoint of $J$. Here, we consider (5.5) on $\mathbf{R}$ and that the data $d_{0}$ has a single facet, hence the same will hold for the solution. We have to check the existence of a solution for all $x \in \mathbf{R}$ for all $t \in[0, T]$. Here, the limitations arise from the constructions of the free boundary $l_{0}$ performed in [30, Section 3.1]. We have already mentioned that the construction essentially depends upon the sign of $\Sigma_{0}$, but it is local in the sense that it uses the data from a neighborhood of $l_{00}$.

Thus, we have to make sure that we can solve $(5.5)_{2}$, that is,

$$
d_{t}(t, x)=\sigma(t, x), \quad d(0, x)=d_{0}(x)
$$

for all large $x$, for example $x>A>l_{00}$ for a constant $A$ and all $t \in[0, T]$. This problem can be solved for all $x \geqq l_{00}$ uniformly in $t>0$,

$$
d(t, x)=\int_{0}^{t} \sigma(s, x) \mathrm{d} s+d_{0}(x)
$$

since we assumed that $\sigma_{x} \in C\left(\mathbf{R}_{+} \times \mathbf{R}\right)$. Moreover, the solution will be Lipschitz continuous if for all $t \geqq 0$ we have that $\operatorname{Lip}(\sigma(t, \cdot)) \leqq L$.

We notice that for all $t>0$ function $\bar{d}(t, \cdot)$ is not only strictly increasing in $x$, but also the derivative $d_{x}(t, x)$ is positive for all $x>l_{00}$.

We also have to check that the Cahn-Hoffman vector $\xi$, specified in the statements of Theorems 10 and 11 , is a unique minimizer of $\mathcal{E}$. This task is left to the reader. Hence, $\left(\Gamma(d(t, \cdot), \xi(t, \cdot))_{t \in[0, T)}\right.$ is a variational solutions. 
Remark 7. We notice that the same kind of the argument shows that Theorem 10 and 11 are valid also if $\sigma=\sigma\left(x_{1}, x_{2}\right)$ satisfies an extension of condition (5.7) for functions of two variables, that is

$$
\sigma\left( \pm x_{1}, \pm x_{2}\right)=\sigma\left(x_{1}, x_{2}\right), \quad \frac{\partial \sigma}{\partial x_{i}}\left(x_{1}, x_{2}\right) x_{i}>0 \text { for } \quad x_{i} \neq 0 .
$$

Moreover, by Remark 3, the Comparison Principle (Theorem 7) holds in this case, too.

\section{References}

1. Angenent, S.B., Gurtin, M.E.: Multiphase thermomechanics with interfacial structure 2 Evolution of an isothermal interface. Arch. Ration. Mech. Anal. 108, 323-391 (1989)

2. Bellettini, G., Caselles, V., Chambolle, A., Novaga, M.: Crystalline mean curvature flow of convex sets. Arch. Ration. Mech. Anal. 179, 109-152 (2006)

3. Bellettini, G., Goglione, R., Novaga, M.: Approximation to driven motion by crystalline curvature in two dimensions. Adv. Math. Sci. Appl., 10, 467-493 (2000)

4. Bellettini, G., Novaga, M.: Approximation and comparison for non-smooth anisotropic motion by mean curvature in $\mathbf{R}^{N}$. Math. Mod. Meth. Appl. Sc. 10, 1-10 (2000)

5. Bellettini, G., Novaga, M., Paolini, M.: Facet-breaking for three-dimensional crystals evolving by mean curvature. Interfaces Free Bound. 1, 39-55 (1999)

6. Bellettini, G., Novaga, M., Paolini, M.: Characterization of facet breaking for nonsmooth mean curvature flow in the convex case. Interfaces and Free Boundaries $\mathbf{3}$, 415-446 (2001)

7. Bellettini, G., Novaga, M., Paolini, M.: On a crystalline variational problem, part I: first variation and global $L^{\infty}$-regularity. Arch. Ration. Mech. Anal. 157, 165-191 (2001)

8. Bellettini, G., Novaga, M., Paolini, M.: On a crystalline variational problem, part II: $B V$ regularity and structure of minimizers on facets. Arch. Ration. Mech. Anal. 157, 193-217 (2001)

9. Chambolle, A., Novaga, M.: Existence and uniqueness for planar anisotropic and crystalline curvature flow. Advanced Studies in Pure Math. (to appear)

10. Chen, Y.-G., Giga, Y., Goto, S.: Uniqueness and existence of viscosity solutions of generalized mean curvature flow equations. J. Differ. Geom. 33, 749-786 (1991)

11. Crandall, M., IshiI, H., Lions, P.-L.: User's guide to viscosity solutions of second order partial differential equations. Bull. Am. Math. Soc. 27, 1-67 (1992)

12. Elliott, C.M., Gardiner, A., Schätzle, R.: Crystalline curvature flow of a graph in a variational setting. Adv. Math. Sci. Appl. 8, 425-460 (1998)

13. Evans, L.C., Spruck, J.: Motion of level sets by mean curvature, I. J. Differential Geom. 33, 635-681 (1991)

14. FukUi, T., Giga, Y.: Motion of a graph by nonsmooth weighted curvature. In: LAKSHmikantham, V. (ed.) World Congress of Nonlinear Analysts '92, pp. 47-56. Walter de Gruyter, Berlin, 1996

15. Giga, Y.: Singular diffusivity-facets, shocks and more. In: Hill, J.M., Moore, R. (eds.) Applied Math Entering the 21st Century. ICIAM 2003 Sydney, pp. 121-138. SIAM, Philadelphia, 2004

16. Giga, Y.: Surface Evolution Equations-A Level Set Approach. Birkhäuser, Basel, 2006

17. Giga, M.-H., Giga, Y.: Evolving graphs by singular weighted curvature. Arch. Ration. Mech. Anal. 141, 117-198 (1998)

18. Giga, M.-H., Giga, Y.: A subdifferential interpretation of crystalline motion under nonuniform driving force. In: CHEN, W.-X., Hu, S.-C. (eds.) In: Proceedings of the International Conference in Dynamical Systems and Differential Equations, Springfield Missouri, 1996, Dynamical Systems and Differential Equations, vol. 1. Southwest Missouri University, Missouri, pp. 276-287, 1998 
19. Giga, M.-H., Giga, Y.: Remarks on convergence of evolving graphs by nonlocal curvature. In: Amann, H., Bandle, C., Chipot, M., Conrad, F., Shafrir, L. (eds.) Progress in Partial Differential Equations, vol. 1. Pitman Research Notes in Mathematics Series, vol. 383, pp. 99-116. Longman, Essex, 1998

20. Giga, M.-H., Giga, Y.: Stability for evolving graphs by nonlocal weighted curvature. Commun. Partial Differ. Equ. 24, 109-184 (1999)

21. Giga, M.-H., Giga, Y.: Generalized motion by nonlocal curvature in the plane. Arch. Ration. Mech. Anal. 159, 295-333 (2001)

22. Giga, M.-H., Giga, Y.: A PDE approach for motion of phase-boundaries by a singular interfacical energy. In: FunAKI, F., OsADA, H. (eds.) Stochastic Analysis on Large Scale Interacting Systems, Advanced Studies in Pure Math., vol. 39, pp. 212-232. Mathematical Society of Japan, 2004

23. Giga, M.-H., Giga, Y.: Very singular diffusion equations: second and fourth order problems. Japan J. Ind. Appl. Math. 27, 323-345 (2010)

24. Giga, M.-H., Giga, Y., Коваyashi, R.: Very singular diffusion equations. In: Maruyama, M., Sunada, T. (eds.) Taniguchi Conference on Mathematics, Nara '98. Adv. Studies in Pure Math., vol. 31, pp. 93-125. Mathematical Society of Japan, 2001

25. Giga, M.-H., Giga, Y., Nakayasu, A.: On general existence results for one-dimensional singular diffusion equations with spatially inhomogeneous driving force. In: CHAMBOLle, A., et al. (eds.) Proceedings of ERC Workshop on "Geometric Partial Differential Equations”, CRM Series 15, pp. 143-170. Ennio De Giorgi Mathematical Research Center, Pisa, 2013

26. Giga, Y., Goto, S., Ishi, H., SAto, M.-H.: Comparison principle and convexity preserving properties for singular degenerate parabolic equations on unbounded domains. Indiana Univ. Math. J. 40, 443-470 (1991)

27. Giga, Y., GóRKa, P., RybKa, P.: Nonlocal spatially inhomogeneous Hamilton-Jacobi equation with unusual free boundary. Discrete Contin. Dyn. Syst. 26, 493-519 (2010)

28. Giga, Y., Gurtin, M.-E., Matias, J.: On the dynamics of crystalline motions. Jpn J. Ind. Appl. Math. 15, 7-50 (1998)

29. Giga, Y., RybKa, P.: Facet bending in the driven crystalline curvature flow in the plane. J. Geom. Anal. 18, 109-147 (2008)

30. Giga, Y., RybKa, P.: Facet bending driven by the planar crystalline curvature with a generic nonuniform forcing term. J. Differ. Equ. 246, 2264-2303 (2009)

31. Hörmander, L.: Notions of Convexity. Birkhäuser, Boston, 1994

32. Kielak, K., Mucha, P.B., RybKa, P.: Almost classical solutions to the total variation flow. J. Evol. Equ. 13, 21-49 (2013)

33. Kinderlehrer, D., Stampacchia, G.: An Introduction to Variational Inequalities and their Applications. Academic Press, New York, 1980

34. Mucha, P.B., RyвкA, P.: A caricature of a singular curvature flow in the plane. Nonlinearity 21, 2281-2316 (2008)

35. Rodrigues, J.F.: Obstacle Problems in Mathematical Physics. Elsevier, Amsterdam, 1987

36. Roosen, A.R.: Crystalline curvature and flat flow in a diffusion field. Unpublished note, 1994

37. Schwartz, L.: Théorie des distributions. Publications de l'Institut de Mathématique de l'Université de Strasbourg. Hermann, Paris, 1966

38. TAYLOR, J.: Constructions and conjectures in crystalline nondifferential geometry. In: Lawson, B., Tanenblat, K. (eds.) Differential geometry, Proceedings of the Conference on Differential Geometry, Rio de Janeiro, Pitman Monographs in Pure and Applied Math., vol. 52, pp. 321-336. Pitman, London, 1991 


\section{Graduate School of Mathematical Sciences \\ University of Tokyo \\ Komaba 3-8-1, Tokyo153-8914, Japan \\ e-mail: mihogiga@ms.u-tokyo.ac.jp e-mail: labgiga@ms.u-tokyo.ac.jp \\ and}

Institute of Applied Mathematics and Mechanics

Warsaw University

ul. Banacha 2, 02-097 Warsaw, Poland

e-mail: rybka@mimuw.edu.pl

(Received August 3, 2012 / Accepted June 24, 2013)

Published online September 24, 2013 - (C) The Author(s) (2013)

This article is published with open access at Springerlink.com 